\title{
Superconformal blocks for mixed 1/2-BPS correlators with SU(2) R-symmetry
}

\author{
Florent Baume, ${ }^{a}$ Michael Fuchs $^{a}$ and Craig Lawrie ${ }^{b}$ \\ ${ }^{a}$ Instituto de Física Teórica UAM-CSIC, \\ Cantoblanco, 28049 Madrid, Spain \\ ${ }^{b}$ Department of Physics and Astronomy, University of Pennsylvania, \\ Philadelphia, PA 19104, U.S.A. \\ E-mail: florent.baume@uam.es, michael.fuchs@uam.es, \\ craig.lawrie1729@gmail.com
}

ABSTRACT: For SCFTs with an SU(2) R-symmetry, we determine the superconformal blocks that contribute to the four-point correlation function of a priori distinct half-BPS superconformal primaries as an expansion in terms of the relevant bosonic conformal blocks. This is achieved by using the superconformal Casimir equation and the superconformal Ward identity to fix the coefficients of the bosonic blocks uniquely in a dimensionindependent way. In addition we find that many of the resulting coefficients are related through a web of linear transformations of the conformal data.

Keywords: Conformal Field Theory, Extended Supersymmetry

ArXiv ePrint: 1908.02768 


\section{Contents}

1 Introduction 1

2 Structure of four-point functions in (S)CFTs 4

2.1 Four-point functions of $\mathcal{D}$-type superconformal primaries 5

$\begin{array}{lll}2.2 & \text { R-symmetry variables } & 7\end{array}$

3 Constraints on four-point functions of half-BPS primaries 9

$\begin{array}{ll}3.1 \text { The superconformal casimir equation } & 10\end{array}$

3.2 The Ward identity 13

$\begin{array}{lll}3.3 & \text { Crossing symmetry constraints } & 15\end{array}$

4 Superconformal blocks of mixed $\mathcal{D}$-type four-point functions $\quad 16$

$\begin{array}{lll}5 & \text { Conclusions } & 21\end{array}$

$\begin{array}{ll}\text { A The superconformal group } & 23\end{array}$

$\begin{array}{ll}\text { B Conformal frame and } \mathrm{SU}(2)_{R} \text { harmonics } & 24\end{array}$

$\begin{array}{ll}\text { C Casimirs and Jack polynomials } & 27\end{array}$

$\begin{array}{ll}\text { D List of coefficients for superconformal blocks } & 29\end{array}$

\section{Introduction}

The use of the Operator Product Expansion (OPE) has shown to be a very powerful tool to study Conformal Field Theories (CFTs). Being convergent [1, 2], it allows for a reduction of any $n$-point correlation function to a function depending solely on the kinematic and three-point function data. In particular, the four-point function of scalar fields is expanded in a series of so-called bosonic conformal blocks, depending only on conformal invariants. These blocks satisfy a second order differential equation, the Casimir equation, from which an explicit form of the blocks can be derived in even spacetime dimensions [3, 4].

With the advent of the numerical bootstrap [5], see [6-9] for reviews, there has been a renewed interest in the study of conformal blocks. Recent progress built on earlier works in two dimensions [10-18] and the associativity property of the OPE to find bounds on the conformal data of given unitary theories. The case where the CFT is endowed with extended supersymmetry is furthermore of particular interest: the possible superconformal multiplets follow a strict classification $[19,20]$ and there exists a subset of these multiplets whose conformal dimensions are fixed by unitarity. For such multiplets, the shortening 
conditions greatly simplify the structure of the conformal blocks. With eight Poincaré supercharges and an R-symmetry group containing $\mathrm{SU}(2)_{R}$, results are known for conformal blocks of four-point functions involving half-BPS operators in $3 D, \mathcal{N}=4[21,22], 4 D, \mathcal{N}=$ $2[23,24], 5 D, \mathcal{N}=1[25]$ and $6 D, \mathcal{N}=(1,0)[26,27]$.

One of the most commonly studied operators for theories with eight supercharges is the momentum map, the short multiplet containing flavour currents. ${ }^{1}$ This enables one to find bounds on some of the conformal data for SCFTs with flavour [23, 25, 26], where there are strong indications that the SCFTs saturating these bounds have known string theory constructions. Therefore the conformal bootstrap might shed some light on the relation between SCFTs and the compactification geometries. For applications to M-theory, see e.g. $[29,30]$.

There are two main paths usually followed to find an explicit form of these blocks. The first is to consider the supersymmetric Ward identity [31-33], which is the most commonly used method. The second is to solve directly the supersymmetric version of the Casimir differential equation $[27,34]$ in a fashion analogous to the method employed to find the bosonic blocks. Both these methods allow one to formally treat the spacetime dimension as a continuous parameter. In this work, we extend previous results about superconformal blocks of four-point functions of scalar superconformal primaries falling in half-BPS, or so-called $\mathcal{D}$-type, multiplets to more general settings. We do so for theories with $\mathrm{SU}(2)$ R-symmetry; without any a priori assumption on the particular R-charges of the external scalars; and in a dimension-independent way, as long as $2<d \leq 6{ }^{2}$

Our focus on theories with R-symmetry algebras isomorphic to $\mathfrak{s u}(2)$ lies in the fact that it corresponds to that of six-dimensional $\mathcal{N}=(1,0)$ SCFTs. These theories are quite special: first thought not to exist, it was discovered that they were related to six-dimensional tensionless strings [35, 36], and it has since been observed that they serve as "master theories" for a host of CFTs in lower dimensions, for example this is the ethos behind the class $\mathcal{S}$ theories that appeared in [37]. While there is no reason why supersymmetry should be imposed, there are no known interacting non-supersymmetric six-dimensional SCFTs and none of them have a known Lagrangian description. Theories in six dimensions therefore offer a very nice playground to study non-perturbative effects and relations to string theory, for instance their connection to the swampland program [38].

As six is the largest dimension allowing for a superconformal algebra [39], $\mathcal{N}=(1,0)$ representation theory provides an overarching language encompassing lower dimensions via dimensional reduction. ${ }^{3}$ Note that in lower than six dimensions the R-symmetry group might enhance due to the transverse directions in the dimensional reduction, such as the extra $U(1)$ factor in four dimensions. We will focus here on multiplets that are uncharged

\footnotetext{
${ }^{1}$ Note that in four dimensions with $\mathcal{N}=2$, there are additional subtleties due to a protected subsector associated to a two-dimensional chiral algebra. [28]

${ }^{2}$ We exclude $d \leq 2$ as in that case some of the generators may decouple from the superconformal algebra, see $[27]$.

${ }^{3}$ We note that for $d \leq 4$, the nomenclature we are using here might not match the one the reader is familiar with. For instance, in four dimensions, the classification of half-BPS multiplets is refined into Higgs and Coulomb type, commonly denoted $\mathcal{E}_{r}$ and $\hat{\mathcal{B}}_{R}$ respectively [40]. We refer to [20] for a dictionary between the $6 \mathrm{D}$ notation and lower dimensions.
} 
under possible additional groups so we can treat them homogenously across dimensions. We refer the reader to $[27,34]$ for more details. Let us review the possible multiplets allowed in theories with $\mathrm{SU}(2)_{R} \mathrm{R}$-symmetry $[19,20]$. There can exist states which are annihilated by a subset of the supercharges. These null states must be absent in unitary theories, and lead to what are referred as short multiplets, as opposed to long multiplets, which do not have null states. It is standard to write long multiplets as $\mathcal{L}\left[\Delta, \ell, J_{R}\right]$, where $\Delta$ is the conformal dimension of the superconformal primary, $\ell$ denotes how the superconformal primary transforms as a traceless-symmetric ${ }^{4}$ representation of $\mathfrak{s o}(1, d-1)$ rotations of the Poincaré algebra, and $J_{R}$ is the charge under $\mathrm{SU}(2)_{R}$.

The different short multiplets are denoted as the $\mathcal{A}-, \mathcal{B}_{-}, \mathcal{C}_{-}$, and $\mathcal{D}$-type multiplets. Unitarity gives lower bounds on the allowed conformal dimensions, $\Delta$, of the superconformal primaries of long multiplets, and is moreover strong enough to completely fix the conformal dimension of the short multiplets as a function of the other group theoretical data and the spacetime dimension. The superconformal multiplets can be summarised as follows:

$$
\begin{aligned}
\mathcal{L}\left[\Delta, \ell, J_{R}\right]: & \Delta>2 \varepsilon J_{R}+\ell+\mu, \\
\mathcal{A}\left[\ell, J_{R}\right]: & \Delta=2 \varepsilon J_{R}+\ell+4 \varepsilon-2, \\
\mathcal{B}\left[\ell, J_{R}\right]: & \Delta=2 \varepsilon J_{R}+\ell+2 \varepsilon, \\
\mathcal{C}\left[J_{R}\right]: & \Delta=2 \varepsilon J_{R}+2, \\
\mathcal{D}\left[J_{R}\right]: & \Delta=2 \varepsilon J_{R},
\end{aligned}
$$

with $\varepsilon=(d-2) / 2, \mu=2 \varepsilon$ for $2<d \leq 4$ and $\mu=4 \varepsilon-2$ for $4 \leq d \leq 6$. We stress again that this corresponds to the standard notation for $d=6$. Indeed, $\mathcal{A}$-type multiplets correspond to the unitarity bound of long multiplets, which for $d \leq 4$ coincides with the bound of type $\mathcal{B}$. Type $\mathcal{C}$ is unique to six dimensions and can be traced back to the presence of self-dual two-forms. Moreover, type $\mathcal{C}$ and $\mathcal{D}$ will appear only with $\ell=0$, since in this work we are restricting ourselves, without loss of generality, to only multiplets in traceless-symmetric representations of the Poincaré algebra.

Some of the short multiplets may already be familiar to the reader: $\mathcal{D}[1 / 2]$ and $\mathcal{C}[0]$ correspond to free hyper- and tensor multiplets respectively, $\mathcal{B}[0,0]$ contains the energy momentum tensor, while $\mathcal{D}[1]$ is the momentum map discussed above, containing the conserved currents associated to possible flavour symmetries [19, 20].

This article is structured as follows: in section 2 we shortly review the decomposition of four-point functions as series of superconformal blocks written in terms of bosonic blocks, differentiating between two possible approaches. One uses a decomposition involving projectors onto irreducible representations of $\mathrm{SU}(2)_{R}$, while the other introduces auxiliary variables for the R-symmetry. In section 3 we discuss constraints the blocks must satisfy, and how one can extract selection rules for the allowed multiplets. More precisely,

\footnotetext{
${ }^{4}$ In this paper we will consider only multiplets that have a superconformal primary in a tracelesssymmetric representation of the Poincaré group as these will be the only contributions to the superconformal blocks that we consider. For a general superconformal multiplet one should replace $\ell$ with an arbitrary representation of $\mathfrak{s o}(1, d-1)$.
} 
we show how the Casimir equation encodes two different types of constraint; and how the Ward identity has to be modified to take into account different external fields. We also comment about the crossing symmetry these correlators must satisfy. Section 4 solves these constraints and discusses some properties of the blocks and their coefficients. We give our conclusions in section 5. In the appendices we discuss our conventions for the superconformal group, how to derive the $\mathrm{SU}(2)_{R}$ harmonics and the Casimir differential operators. We also review various relations satisfied by the Jack polynomials, and give a non-exhaustive list of the coefficients of the superconformal blocks. In addition, we provide a Mathematica file as supplementary material containing an exhaustive list of the coefficients for all the superconformal blocks that appear in the four-point functions of $\frac{1}{2}$-BPS scalar operators.

\section{Structure of four-point functions in (S)CFTs}

In this section, we review the structure imposed by conformal invariance on four-point functions of (super)conformal primaries. We start by recalling the non-supersymmetric results to set our notation and conventions, and then move to the case of superconformal primaries of $\mathcal{D}$-type multiplets. In that case we will present two different - but equivalent - approaches, namely a decomposition in terms of projectors of the R-symmetry, and one involving an auxiliary variable.

In the non-supersymmetric case, the four-point functions of four a priori different conformal scalar primaries, $\phi_{i}$, of conformal dimension, $\Delta_{i}$, is well known to admit a decomposition in term of bosonic conformal blocks $, g_{\Delta, \ell}^{\Delta_{12}, \Delta_{34}},[3,4,41]$

$$
\left\langle\phi_{1}\left(x_{1}\right) \phi_{2}\left(x_{2}\right) \phi_{3}\left(x_{3}\right) \phi_{4}\left(x_{4}\right)\right\rangle=K_{4} \sum_{\mathcal{O}} \lambda_{12 \mathcal{O}} \lambda_{34 \mathcal{O}} g_{\Delta, \ell}^{\Delta_{12}, \Delta_{34}}(u, v) .
$$

The sum is taken over all conformal primaries, $\mathcal{O}$, with conformal data, $(\Delta, \ell)$, allowed in the OPEs, and $\lambda_{i j \mathcal{O}}$ corresponds to the coefficient of the three-point function, $\left\langle\phi_{i} \phi_{j} \mathcal{O}\right\rangle$. Moreover, the kinematic prefactor will depend on the conformal dimensions of the external primaries, $\Delta_{i}$, and can be shown to take the general form

$$
K_{4}=\frac{1}{\left(x_{12}^{2}\right)^{\frac{\Delta_{1}+\Delta_{2}}{2}}\left(x_{34}^{2}\right)^{\frac{\Delta_{3}+\Delta_{4}}{2}}}\left(\frac{x_{24}^{2}}{x_{14}^{2}}\right)^{\frac{\Delta_{12}}{2}}\left(\frac{x_{14}^{2}}{x_{13}^{2}}\right)^{\frac{\Delta_{34}}{2}},
$$

where

$$
x_{i j}=\left|x_{i}-x_{j}\right|, \quad \text { and } \quad \Delta_{i j}=\Delta_{i}-\Delta_{j} .
$$

The blocks are invariant under conformal transformation and therefore depend on the two independent invariant cross-ratios, defined by

$$
u=\frac{x_{12}^{2} x_{34}^{2}}{x_{13}^{2} x_{24}^{2}}=z \bar{z}, \quad v=\frac{x_{14}^{2} x_{23}^{2}}{x_{13}^{2} x_{24}^{2}}=(1-z)(1-\bar{z}) .
$$

We have directly defined the two common variables, $(z, \bar{z})$, that will be convenient throughout this work. We note that while in Euclidean space these are complex conjugate, they are independent real variables for Lorentzian signature. 
The conformal blocks satisfy various properties related to crossing symmetries of the four-point function, and can be computed as the solution of a partial differential equation, dubbed the Casimir equation. We delay a discussion of these properties to section 3 , where we will delve into more details.

Here, we are interested in superconformal theories, and imposing supersymmetry on top of conformal invariance will act as selection rules for the OPE in two ways: first, the R-symmetry plays the role of a flavour symmetry, restricting the possible representations allowed in the OPE of the external primaries; second, the bosonic blocks will rearrange themselves into superconformal blocks whose structure is compatible with superconformal representation theory.

The remainder of this section is dedicated to outline the selection rules and structure of four-point functions of SCFTs with eight supercharges and a R-symmetry algebra isomorphic to $\mathfrak{s u}(2)_{R}$.

\subsection{Four-point functions of $\mathcal{D}$-type superconformal primaries}

Let us now focus on the four-point function of superconformal primaries of a $\mathcal{D}$-type multiplet belonging to an SCFT with SU(2) R-symmetry. As reviewed in the introduction, these multiplets, denoted $\mathcal{D}\left[J_{R}\right]$, are half-BPS and fall into the spin- $J_{R}$ representation of the R-symmetry group. ${ }^{5}$ These multiplets obey a shortening condition that relates the conformal dimension of their superconformal primary to the R-charge [19, 20, 42]:

$$
\Delta=2 \varepsilon J_{R}, \quad \varepsilon=\frac{d-2}{2} .
$$

The spacetime dimension, $d$, is left arbitrary and as we will see most of the expressions we will deal with are valid for any $d$.

The spin- $J$ representation of $\mathrm{SU}(2)$, with $J \in \frac{1}{2} \mathbb{N}$, is an irreducible representation that can be constructed as the $2 J$ th symmetric tensor power of the fundamental representation, 2 , that is

$$
\operatorname{Sym}^{2 J} \mathbf{2}=\mathbf{2} \boldsymbol{J}+\mathbf{1}
$$

where, as usual, boldface denotes an irreducible representation by its dimension. We can realise an operator that transforms in this way by introducing $2 J$ symmetric fundamental indices

$$
\mathcal{O}^{\left(\alpha_{1} \ldots \alpha_{2 J}\right)}(x), \quad \alpha_{i}=1,2,
$$

which are raised and lowered by the usual Levi-Civita tensor, $\varepsilon_{\alpha \beta}$, and $\left(\alpha_{1} \ldots \alpha_{n}\right)$ indicates the symmetrisation of the indices. Alternatively, one can introduce an index, $M$, which runs over the spins of the $\mathbf{2} \boldsymbol{J}+\mathbf{1}$ representation in which the operator is transforming. The spins in the representation are $M=J, J-1, \cdots,-J$. We will prefer here the latter notation, $\mathcal{O}^{M}(x)$, when we consider a scalar transforming in the spin- $J$ representation of the $\mathrm{SU}(2)$ R-symmetry.

\footnotetext{
${ }^{5}$ Notice that e.g. $[19,20]$ use Dynkin indices to label the R-charge, which are integer valued. We choose to use the spin notation - half-integer labels - in order to unclutter many expressions.
} 
Let $\phi_{i}^{M_{i}}$ be the superconformal primary of any half-BPS superconformal multiplet, $\mathcal{D}\left[J_{i}\right]$, with conformal dimension, $\Delta_{i}$, set by equation (2.5). The correlation function of four of these primaries is severely constrained by symmetry. First, as we saw in the nonsupersymmetric case, conformal symmetry fixes the spacetime dependence up to a function of the invariant cross-ratios, $u, v$, and a kinematic term that can be factored out:

$$
\left\langle\phi_{1}^{M_{1}}\left(x_{1}\right) \phi_{2}^{M_{2}}\left(x_{2}\right) \phi_{3}^{M_{3}}\left(x_{3}\right) \phi_{4}^{M_{4}}\left(x_{4}\right)\right\rangle=K_{4} F^{M_{1} M_{2} M_{3} M_{4}}(u, v) .
$$

Treating the R-symmetry as a flavour symmetry, the four-point function must be an invariant tensor of $\mathrm{SU}(2)$, and therefore $F^{M_{1} M_{2} M_{3} M_{4}}(u, v)$ is an invariant under both conformal symmetry and $\mathrm{SU}(2)_{R}$. Using the OPE, this function can be expanded into contributions coming from each of the superconformal multiplets, $\chi$, allowed in the expansion,

$$
F^{M_{1} M_{2} M_{3} M_{4}}(u, v)=\sum_{\chi} \lambda_{12 \chi} \lambda_{34 \chi} \mathcal{G}_{\chi}^{M_{1} M_{2} M_{3} M_{4}}(u, v)
$$

Inside of each superconformal multiplet there are primary operators that transform in different representations of the SU(2) R-symmetry. By introducing projectors on the spin$J$ representation, $P_{J}^{M_{1} M_{2} M_{3} M_{4}}$, in the superconformal block, $\mathcal{G}_{\chi}^{M_{1} M_{2} M_{3} M_{4}}$, we can further split the four-point function into a sum over all allowed R-symmetry channels. Thus for each superconformal multiplet we can expand as

$$
\mathcal{G}_{\chi}^{M_{1} M_{2} M_{3} M_{4}}=\sum_{J \in \mathcal{J}} P_{J}^{M_{1} M_{2} M_{3} M_{4}} \mathcal{G}_{\chi}^{J}(u, v),
$$

where $\mathcal{J}$ is the set of all allowed propagating spins. By considering the $s$-channel for the OPE one can see that the set of $\mathrm{SU}(2)$ representations that correspond to the propagating spins is determined by the tensor products

$$
\left(\left(2 J_{1}+1\right) \otimes\left(2 J_{2}+1\right)\right) \cap\left(\left(2 J_{3}+1\right) \otimes\left(2 J_{4}+1\right)\right) .
$$

Recalling how $\mathrm{SU}(2)$ tensor products decompose, one can easily compute the set of propagating spins $\mathcal{J}$ for any given $J_{1}, \cdots, J_{4}$. This can be written as

$$
\mathcal{J}=\left\{\operatorname{Max}\left(\left|J_{2}-J_{1}\right|,\left|J_{4}-J_{3}\right|\right), \ldots, \operatorname{Min}\left(J_{1}+J_{2}, J_{3}+J_{4}\right)\right\},
$$

where we add the caveat that the set is empty if the start and end values different by $n+\frac{1}{2}$ for some integer $n$ - in such a case there are no propagating spins. To give an explicit example, for coinciding representations, $J=J_{i}$, then we have

$$
\mathcal{J}=\{2 J, 2 J-1, \cdots, 0\}
$$

as the propagating spins appearing in the sum in (2.10).

Finally, the superconformal blocks can be decomposed into bosonic blocks, where by symmetry only the bosonic components of a superconformal multiplet can contribute to the OPE. In fact, since we are considering OPEs of scalar fields, only fields that are symmetric and traceless are allowed. Therefore the spin- $J$ part of superconformal block, 
$\mathcal{G}_{\chi}^{J}(u, v)$, associated to the superconformal multiplet can be written as a sum over bosonic conformal blocks, $g_{\Delta, \ell}^{\Delta_{12}, \Delta_{34}}(u, v)$, and collects the contribution of all the constituent (nonsupersymmetric) primary fields of the superconformal multiplet that have the aforementioned spin, $J$ :

$$
\mathcal{G}_{\chi}^{J}(u, v)=\sum_{(\tilde{\Delta}, \tilde{\ell}) \in \chi} f_{\tilde{\Delta}, \tilde{\ell}}^{J} g_{\tilde{\Delta}, \tilde{\ell}}^{\Delta_{12}, \Delta_{34}}(u, v),
$$

where $(\tilde{\Delta}, \tilde{\ell})$ correspond to the data of the relevant superconformal descendants inside the multiplet with fixed R-charge, $J$. Finding the explicit expression for a superconformal block therefore reduces to determining the coefficients $f_{\Delta, \ell}^{J}$. In order to do so, we will use both the Casimir equation and Ward identity to constrain them, and eventually fix them all in terms of the data of the superconformal primary.

The full superconformal block associated to a multiplet, $\chi$, whose primary has conformal data $\left(\Delta, \ell, J_{R}\right)$ can therefore be decomposed into a sum over all $\mathcal{G}_{\chi}^{J}$. We note that throughout this paper the labelling $\left(\Delta, \ell, J_{R}\right)$ will always denote the data of the superconformal primary of a given superconformal multiplet, $\chi$, while $(\tilde{\Delta}, \tilde{\ell}, J)$ will refer to that of any of its states, including the primary. In the case of eight supercharges, one can show that the allowed R-charges inside a multiplet are between $J_{R}-2$ and $J_{R}+2$. Furthermore each application of a supercharge will raise the conformal dimension by $\frac{1}{2}$, and possibly change its Poincaré representation, thus the superconformal block can be written as

$$
\mathcal{G}_{\chi}^{M_{1} M_{2} M_{3} M_{4}}=\sum_{J=J_{R}-2}^{J_{R}+2} \sum_{m=0}^{4} \sum_{n=-2}^{2} P_{J}^{M_{1} M_{2} M_{3} M_{4}} f_{\Delta+m, \ell+n}^{J} g_{\Delta+m, \ell+n}^{\Delta_{12}, \Delta_{34}}(u, v) .
$$

Of course, depending on the type of multiplet considered and its content, not all $f_{\Delta, \ell}^{J}$ are non-vanishing, and some of them can be set to zero by group theoretical arguments. We will further expand on the structure of superconformal multiplets in section 3 .

\section{$2.2 \quad$ R-symmetry variables}

It is sometimes useful to introduce auxiliary variables, $Y^{\alpha}$, to encode R-symmetry transformations in a more convenient way $[32,33]$. These variables can be used to contract all possible R-symmetry indices of a given operator,

$$
\mathcal{O}(x, Y)=Y_{\alpha_{1}} \cdots Y_{\alpha_{2 J}} \mathcal{O}^{\alpha_{1} \ldots \alpha_{2 J}}(x),
$$

such that if $\mathcal{O}(x)$ is a scalar primary of conformal dimension, $\Delta$, in the spin- $J$ representation, $\mathcal{O}(x, Y)$ is a homogeneous function of degree $(-\Delta, 2 J)$.

In the case of four-point functions of $\mathcal{D}$-type primaries, the discussion at the beginning of this section has to be modified to take into account auxiliary variables, and an additional prefactor related to $Y^{\alpha}$ can be extracted from the correlator,

$$
\left\langle\phi_{1}\left(x_{1}, Y_{1}\right) \phi_{2}\left(x_{2}, Y_{2}\right) \phi_{3}\left(x_{3}, Y_{3}\right) \phi_{4}\left(x_{4}, Y_{4}\right)\right\rangle=K_{4} K_{4}^{R} F(u, v ; w) .
$$

The quantity $K_{4}$ is the usual kinematic prefactor given by equation (2.2), while $K_{4}^{R}$ takes into account the homogeneity of the four-point function with respect to $Y^{\alpha}$.

$$
\begin{aligned}
K_{4}^{R}= & \left(Y_{12}\right)^{a_{1}}\left(Y_{13}\right)^{a_{2}}\left(Y_{14}\right)^{-a_{1}-a_{2}+2 J_{1}}\left(Y_{23}\right)^{-a_{1}-a_{2}+J_{12}^{+}+J_{34}} \\
& \times\left(Y_{24}\right)^{a_{2}-J_{12}-J_{34}}\left(Y_{34}\right)^{a_{1}-J_{12}^{+}+J_{34}^{+}} .
\end{aligned}
$$


where we defined the quantity $J_{i j}=J_{i}-J_{j}$ and $J_{i j}^{+}=J_{i}+J_{j}$. The so-far undefined function in the r.h.s. of (2.17) must be an invariant under both conformal and R-symmetry transformations, and we must therefore find the analogue of the invariant cross-ratios $u, v$ for the R-symmetry. It can be shown that the unique candidate is given by

$$
w=\frac{\left(Y_{1} \cdot Y_{2}\right)\left(Y_{3} \cdot Y_{4}\right)}{\left(Y_{1} \cdot Y_{4}\right)\left(Y_{2} \cdot Y_{3}\right)}, \quad Y_{i j}=Y_{i}^{\alpha} Y_{j}^{\beta} \varepsilon_{\alpha \beta} .
$$

The coefficients, $a_{1}, a_{2}$, in the prefactor are arbitrary constants that effectively rescale $F(u, v ; w)$ by factors of $w$ and $1+w$, respectively. A particular choice for these constants is merely a choice of convention; for more details on the possible choices and how they relate to previous works see appendix B.

In a similar fashion that was reviewed in the case of "uncontracted" fields, the invariant function can be split into contributions from each superconformal multiplet, $\chi$,

$$
F(u, v ; w)=\sum_{\chi} \lambda_{12 \chi} \lambda_{34 \chi} \mathcal{G}_{\chi}(u, v ; w),
$$

and into contributions from the different R-symmetry channels given now by $\mathrm{SU}(2)_{R}$ harmonics,

$$
\mathcal{G}_{\chi}(u, v ; w)=\sum_{J \in \mathcal{J}} \mathcal{P}_{J}^{J_{12}, J_{34}}(w) \mathcal{G}_{J}^{\chi}(u, v) .
$$

The harmonics, $\mathcal{P}_{J}(w)$ are obtained by inserting the quadratic Casimir for $\mathrm{SU}(2)_{R}$ in the four-point function, in a similar way to what is usually done to obtain bosonic conformal blocks,

$$
\mathcal{P}_{J}^{J_{12}, J_{34}}(w)=c_{J} \frac{w^{-J-\left(a_{1}-\left(J_{1}+J_{2}\right)\right)}}{(1+w)^{a_{2}}}{ }_{2} F_{1}\left(-\left(J+J_{12}\right),-\left(J+J_{34}\right) ;-2 J ;-w\right),
$$

and are related to the R-symmetry projectors, $P_{J}^{M_{1} M_{2} M_{3} M_{4}}$, introduced in (2.10). Note that the $\mathrm{SU}(2)_{R}$ harmonics a priori depend on the combination $J_{1}+J_{2}$. Our choice of normalisation, $a_{1}=J_{1}+J_{2}, a_{2}=J_{34}$, absorbs it and leaves the dependence on external data only on the difference $J_{i j}=J_{i}-J_{j}$. The full four-point function does of course not depend on this convention, but it will make some of the intermediate expressions easier. We will comment as to possible differences between conventions when needed.

The hypergeometric function can, in principle, be recast into, perhaps more familiar, Jacobi polynomials [33], but in practical computations we find the hypergeometric function more convenient. We refer to appendix B for additional details on the derivation of (2.22) and possible conventions.

The quantity $c_{J}$ is an arbitrary constant we choose to be

$$
c_{J}=\frac{(-1)^{J} \Gamma(2 J+1)}{\left(1-J_{12}\right)_{J}\left(1-J_{34}\right)_{J}},
$$

such that the spin- $J$ contribution to the superconformal blocks, $\mathcal{G}_{J}^{\chi}$, defined here agrees with those of the "uncontracted" notation when using our choice of normalisation for the projectors (see later in equation (3.16)). Using the expansion (2.14) in terms of bosonic blocks 
one finds the contribution to the four-point function of a superconformal multiplet, $\chi$, is

$$
\mathcal{G}_{\chi}(u, v ; w)=\sum_{J \in \mathcal{J}} \sum_{m, n} f_{\Delta+m, \ell+n}^{J} \mathcal{P}_{J}^{J_{12}, J_{34}}(w) g_{\Delta+m, \ell+n}^{\Delta_{12}, \Delta_{34}}(u, v) .
$$

As the set of different possible superconformal multiplets is known, we can further use the structure of these multiplets as a selection rules for the possible multiplets appearing in the OPE. As we will see in the following sections, the Casimir and Ward identities can in general only be satisfied if all the non-zero bosonic blocks in the decomposition (2.14) are present - in the case of coincident $J_{i}$ some of them are vanishing, but their absence can be traced back to crossing symmetry.

This constrains further the allowed superconformal multiplets in the expansion. Indeed, let us denote the largest spin in (2.12) by $J_{\max }$. A long multiplet whose superconformal primary has R-charge $J_{R}=J_{\max }$ also contains states with $J=J_{\max }+1, J_{\max }+2$. $\mathrm{SU}(2)_{R}$ symmetry prohibits these states to appear in the OPE and, as we will see, the full multiplet is either forbidden to participate, or the structure of the coefficients $f_{\tilde{\Delta}, \tilde{\ell}}^{J>J_{\max }}$ is such that they precisely vanish. In fact, the information about possible null states, e.g. the conservation of flavour currents inside short multiplets $\mathcal{D}[1]$, is also encoded in the structure of the coefficients. The case of $\mathcal{D}$-type multiplets is not plagued by this constraint, as the descendants have an R-charge smaller than that of the superconformal primary, but for the $\mathcal{B}$-type multiplet the presence of a state with $\mathrm{R}$-charge $J_{R}+1$ leads to reduced options.

The last two possible types of short multiplets, $\mathcal{A}$ and $\mathcal{C}$ can be shown to be incompatible with the Ward identity and do not contribute to the four-point function [26, 43]. Schematically, the block decomposition thus takes the form

$$
F(u, v ; w) \sim \sum_{J=J_{\min }}^{J_{\max }-2} \mathcal{L}[\Delta, \ell, J]+\sum_{J=J_{\min }}^{J_{\max }-1} \mathcal{B}[\ell, J]+\sum_{J=J_{\min }}^{J_{\max }} \mathcal{D}[J],
$$

where a sum over all possible $\Delta, \ell$ allowed by unitarity $(1.1)$ is understood. When the R-symmetry group contains an extra factor, such as $\mathcal{N}=4$ in three dimensions where it is semi-simple, $\mathrm{SU}(2) \times \mathrm{SU}(2)$, this selection rule is modified. In particular, for $\mathcal{N}=2$ in four dimensions, the presence of the abelian group, U(1), implies the existence of an extra sector with contributions from superconformal multiplets with a primary in a nontraceless-symmetric representation of the Lorentz group [23].

We end this section by noting that while subsection 2.1 is up to minor modifications similar to the decomposition used in [34] to obtain the blocks of four momentum-map operators, the decomposition with auxiliary variables gets more involved when considering non-coincident operators, and depends heavily on $J_{12}$ and $J_{34}$. When setting them to zero, the $\mathrm{SU}(2)_{R}$ harmonic (2.22) reduces to Legendre polynomials and one recovers the familiar expressions used in e.g. [23, 26].

\section{Constraints on four-point functions of half-BPS primaries}

Before delving into the constraints satisfied by blocks and, by extension, the coefficients $f_{\tilde{\Delta}, \tilde{\ell}}^{J}$, let us recall some facts about the representation theory of the superconformal group 
with extended supersymmetry that will prove useful when computing the superconformal blocks.

Unitary representations of these groups have been extensively studied, starting with [44-46], and more recently with [19, 20, 31, 42, 47, 48]. In addition to the Poincaré generators, there are additional fermionic generators, $Q_{\alpha A}, S^{\alpha A}$ - in our case eight of each - which act in conjunction with $P_{\mu}$ and $K_{\mu}$ as ladder operators. Our convention for the superconformal algebra is set in appendix A.

A superconformal primary, $\mathcal{O}$ with conformal dimension, $\Delta$, R-charge, $J_{R}$, and falling into a traceless-symmetric representation of the Poincaré group ${ }^{6} \ell$, is by definition annihilated by all conformal supercharges, $S^{\alpha A}|\mathcal{O}\rangle=0$, as well as by the special conformal transformation generator, $K_{\mu}|\mathcal{O}\rangle=0$. Using combinations of all (Poincaré) supercharges, $Q_{a \alpha}$, we can reach an additional set of states whose superconformal data are related to that of the superconformal primary. More precisely, applying a supercharge to the superconformal primary will give rise to a state whose conformal dimension has been raised by $\frac{1}{2}$, and whose R-charge and Poincaré representation are changed. Each multiplet contains primary states with, at most, conformal dimension $\Delta+4$, R-charge between $J_{R}-2$ and $J_{R}+2$, and, focusing on traceless-symmetric representations, Poincaré representation between $\ell-2$ and $\ell+2[19,20]$.

Short multiplets are then multiplets for which some descendants are annihilated by given combinations of the supercharges. In particular, the $\mathcal{D}$-type multiplets that are the focus of this work have a primary annihilated by half of the supercharges and are therefore half-BPS states. This property reveals itself crucial when studying their fourpoint functions, as it leads to simplifications that do not occur in the other cases.

This section is dedicated to the constraints satisfied by the superconformal blocks. We will first work out the constraints from the Casimir equations. One will be a differential equation while other will give a selection rule for the multiplets at the boundary of the set of the $\mathrm{SU}(2)_{R}$ representations, $\mathcal{J}$, appearing in the OPE. We will then consider the consequences of the Ward identity when considering the R-symmetry auxiliary variables. This constraint will prove the strongest and will uniquely fix all possible superconformal blocks. Lastly we will outline constraints from crossing symmetry.

\subsection{The superconformal casimir equation}

Having set up the decomposition of the four-point function of $\mathcal{D}$-type superconformal multiplets into conformal blocks, we are now ready to find how to use the Casimir equation to constrain the coefficients of the superconformal blocks and eventually fix some of them. This technique has already been explored in [27, 34] for theories with four and eight supercharges, and this section generalises their results to arbitrary R-charge for SCFTs with eight supercharges.

Before moving to the supersymmetric case, it is useful to recall how the Casimir equation has been used to find the bosonic blocks for arbitrary $\varepsilon$.

\footnotetext{
${ }^{6}$ There are of course multiplets in other representations of the Poincaré group, but these will not contribute to the quantities computed in this work.
} 
The idea is to insert the completeness relation of projectors, $P_{\mathcal{O}}$, and the conformal Casimir, ${ }^{7} C_{\text {bos }}^{2}$, into the correlator to obtain the contribution from every primary, $\mathcal{O}$, and its descendants,

$$
P_{\mathcal{O}}=\sum_{a, b=\left(P_{\mu}\right)^{n} \mathcal{O}, n \geq 0}|a\rangle\langle b|\langle a \mid b\rangle^{-1}, \quad \mathbf{1}=\sum_{\mathcal{O}} P_{\mathcal{O}}
$$

Letting $C_{\text {bos }}^{2}$ act on $\phi_{1} \phi_{2}$ as a first-order linear differential operator, one finds that the bosonic blocks satisfy the differential equation [4],

$$
\mathcal{D}_{\text {bos }} g_{\Delta, \ell}^{\Delta_{12}, \Delta_{34}}(z, \bar{z})=c_{\Delta, \ell}^{\text {bos }} g_{\Delta, \ell}^{\Delta_{12}, \Delta_{34}}(z, \bar{z}) .
$$

The second-order differential operator, $\mathcal{D}_{\text {bos }}$, depends explicitly on the difference between the conformal dimensions of the primaries,

$$
\begin{aligned}
\mathcal{D}_{\text {bos }} & =\mathcal{D}_{z}+\mathcal{D}_{\bar{z}}+4 \varepsilon \frac{z \bar{z}}{z-\bar{z}}\left((1-z) \partial_{z}-(1-\bar{z}) \partial_{\bar{z}}\right) \\
\mathcal{D}_{z} & =2 z^{2}(1-z) \partial_{z}^{2}-\left(2+\Delta_{34}-\Delta_{12}\right) z^{2} \partial_{z}+\frac{\Delta_{12} \Delta_{34}}{2} z
\end{aligned}
$$

Notice that we are considering bosonic blocks in terms of the variables $z, \bar{z}$, rather that the conformal cross-ratios $u, v$. This will make the various differential operators in this section simpler.

The r.h.s. of equation (3.2) depends solely on the quadratic Casimir eigenvalue associated to the bosonic primary with conformal data $(\Delta, \ell)$,

$$
c_{\Delta, \ell}^{\mathrm{bos}}=\Delta(\Delta-2(\varepsilon+1))+\ell(\ell+2 \varepsilon) .
$$

For even dimensions, equation (3.2) simplifies and an analytic expression can be found in terms of hypergeometric functions [4]. For arbitrary $\varepsilon$ the solutions of the Casimir equation are unknown, but there exists rapidly converging power series in terms of radial coordinates $[49,50]$, as well as recursion relations by studying their analytic structure [51-53].

To obtain the supersymmetric version of (3.2) for superconformal blocks, $\mathcal{G}_{\Delta, \ell}^{M_{1} M_{2} M_{3} M_{4}}$, the conformal Casimir, its eigenvalue and projectors are replaced by their supersymmetric cousins [27],

$$
\begin{aligned}
C^{2} & =C_{\mathrm{bos}}^{2}+C_{\mathrm{SUSY}}^{2}+C_{R}^{2}, \\
c_{\Delta, \ell, J_{R}} & =c_{\Delta, \ell}^{\mathrm{bos}}+4 \Delta+2 \varepsilon J_{R}\left(J_{R}+1\right) .
\end{aligned}
$$

The procedure to get the Casimir equation is the same as in the bosonic case, with the exception that the additional generators have to be taken into account:

$$
\left(\mathcal{D}_{\mathrm{bos}}+\mathcal{D}_{\mathrm{SUSY}}+\mathcal{D}_{R}\right) \mathcal{G}_{\chi}^{M_{1} M_{2} M_{3} M_{4}}(z, \bar{z})=c_{\Delta, \ell, J_{R}} \mathcal{G}_{\chi}^{M_{1} M_{2} M_{3} M_{4}}(z, \bar{z}) .
$$

The quadratic Casimir, acting as differential operator, will behave differently depending on the values of $M_{i}$. Note that for arbitrary values of $M_{i}$, inserting the part of the Casimir

\footnotetext{
${ }^{7}$ See appendix (A) for a definition in terms of the generators, and appendix B for a derivation of a Casimir equation in the case of $\mathrm{SU}(2)_{R}$ harmonics.
} 
involving the supercharges, $C_{\mathrm{SUSY}}^{2}$, in the four-point function will produce four-point functions involving mixed scalar and fermionic fields, and the resulting Casimir equations will not be partial differential equations. This makes it challenging at best to compute the Casimir equation in terms of well-defined quantities.

In the case of short $\mathcal{D}$-type superconformal multiplets however, the primaries in the highest and lowest weight representations of the R-symmetry are annihilated by half of the supercharges, $Q_{\alpha A}$, respectively [19, 20, 42],

$$
Q_{1 A} \phi^{J}=0, \quad Q_{2 A} \phi^{(-J)}=0, \quad \forall A .
$$

Therefore when the superconformal block, $\mathcal{G}_{\chi}^{M_{1} M_{2} M_{3} M_{4}}$, involves highest and lowest weights of the $\mathrm{SU}(2)_{R}$ representations, we obtain a well-defined partial differential equation. Without loss of generality, we will henceforth assume that $J_{1} \leq J_{2} \leq J_{3} \leq J_{4}$. This makes the possible values of the set $\mathcal{J}$ more tractable and makes it easier to see when the projectors are vanishing. As shown in appendix C, using the procedure introduced in [34], this leads us to consider two types of correlation functions, which we dub type (I) and (II):

$$
(\mathrm{I}):\left\langle\phi_{1}^{J_{1}}\left(x_{1}\right) \phi_{2}^{J_{2}}\left(x_{2}\right) \phi_{3}^{M_{3}}\left(x_{3}\right) \phi_{4}^{M_{4}}\left(x_{4}\right)\right\rangle, \quad(\mathrm{II}):\left\langle\phi_{1}^{J_{1}}\left(x_{1}\right) \phi_{2}^{M_{2}}\left(x_{2}\right) \phi_{3}^{J_{3}}\left(x_{3}\right) \phi_{4}^{M_{4}}\left(x_{4}\right)\right\rangle \text {. }
$$

The differential operator, $\mathcal{D}_{\mathrm{SUSY}}$, acts differently on the conformal blocks depending on the type of correlation function considered,

$$
\begin{aligned}
(\mathrm{I}) & : \mathcal{D}_{\mathrm{SUSY}}=4\left(\Delta_{1}+\Delta_{2}\right), \\
(\mathrm{II}) & : \mathcal{D}_{\mathrm{SUSY}}=4 z(1-z) \partial_{z}+\bar{z}(1-\bar{z}) \partial_{\bar{z}}-2(z+\bar{z}) \Delta_{34} .
\end{aligned}
$$

In both cases, the differential operator associated to $C_{R}^{2}$ can be obtained simply by applying it on the projector instead of $\phi_{1} \phi_{2}$. It then gives the eigenvalue of the R-charge ${ }^{8}$ of the bosonic block considered,

$$
\mathcal{D}_{R}=-2 \varepsilon J(J+1) .
$$

The different types of correlators will lead to two different types of constraints, which we now outline.

Constraints from Type (I). For the first case, type (I), the only value of $J$ leading to a non-trivial projectors is $J=J_{1}+J_{2}$. This can be seen by writing the projectors in terms of Clebsch-Gordan coefficients. As there is only one contribution in equation (3.8), the projector drops out of the Casimir equation and one obtains

$$
\left(\mathcal{D}_{\text {bos }}-2 \varepsilon\left(J_{1}+J_{2}\right)\left(J_{1}+J_{2}-3\right)\right) \mathcal{G}_{\chi}^{J_{1}+J_{2}}=c_{\Delta, \ell, J_{R}} \mathcal{G}_{\chi}^{J_{1}+J_{2}} .
$$

Decomposing the multiplet in terms of bosonic blocks with conformal data shifted away from the primary, $(\tilde{\Delta}, \tilde{\ell})=(\Delta+m, \ell+n)$, one finds the constraint

$$
(m-2)(2 \Delta+m)+n(2 l+n)+2 \varepsilon\left(J_{R}\left(J_{R}+1\right)-J_{\max }\left(J_{\max }-3\right)+n-m\right)=0 .
$$

\footnotetext{
${ }^{8}$ Note that in the presence of an extra R-symmetry factor, the Casimir gets additional contributions, changing the form of the blocks. In particular, abelian factors may allow for multiplets whose superconformal primary is not in a traceless-symmetric representation of the Lorentz group, see e.g. [34].
} 
This constraint must be satisfied by any states in a given multiplet susceptible to have $J=J_{\max }$, and one must check whether a state is allowed or not on a case-by-case basis. We will do so in section 4 .

Constraints from Type (II). For correlation functions of type (II), the part of the Casimir associated to the supercharges no longer act as multiplication, but involves derivatives with respect to the coordinates $z, \bar{z}$. Moreover the projectors are no longer vanishing and we must consider a sum over all possible R-charges in the set $\mathcal{J}$.

The canonical normalisation for projectors is usually fixed such that the trace of the projector gives the dimension of the representation. Here, we have chosen a slightly different convention that will lead to a simpler expression for the Casimir equations. Indeed, for type (II) correlators, $M_{1}, M_{3}$ are fixed to be the highest weights of their respective representations. The remaining two can be parametrised as the deviation from the lowest weights of $J_{1}$ and $J_{3}, M_{2}=-J_{1}+m, M_{4}=-J_{3}-m$ since, by SU(2) invariance, it is required that $\sum_{i} M_{i}=0$. Note that for coincident external R-charges the only non-vanishing possibility is $m=0$.

As one can check, the four-point functions for any choice of $m$ are proportional to each other. Furthermore for all allowed intermediate R-charges, $J \in \mathcal{J}$, the projectors are non-vanishing if $m$ is chosen in the allowed range. We thus choose our normalisation convention in such a way that all contributions from the projectors are one for $m=0,{ }^{9}$

$$
P_{J}^{J_{1}\left(-J_{1}\right) J_{3}\left(-J_{3}\right)}=1, \quad \forall J \in \mathcal{J} .
$$

In this convention, the type (II) Casimir equation reduces to

$$
\sum_{J \in \mathcal{J}}\left(\mathcal{D}_{\text {bos }}+\mathcal{D}_{\mathrm{SUSY}}-2 \varepsilon J(J+1)-c_{\Delta, \ell, J_{R}}\right) \mathcal{G}_{\chi}^{J}(z, \bar{z})=0 .
$$

Because of the derivative in (3.12), the blocks, superconformal or otherwise, are no longer eigenfunctions of $\mathcal{D}_{\text {SUSY }}$, and constraints can no longer be solved block by block. To overcome this, the usual strategy is to decompose the bosonic blocks in terms of orthogonal Jack polynomials, $P_{\lambda_{1}, \lambda_{2}}^{\varepsilon}[27,34]$, which we will accomplish in section 4.

\subsection{The Ward identity}

In the previous subsection, we obtained constraints on the coefficients, $f_{\tilde{\Delta}, \tilde{\ell}}^{J}$ by letting the Casimir operator act as a differential operator on the four-point function, which differs depending on the type of projectors considered. One can ask whether the same kinds of constraints can be obtained using the auxiliary variables, $Y^{\alpha}$, reviewed in section 2.2. In this case, the constraints are obtained through the Ward identity satisfied by the correlators [32, 33]. For half-BPS multiplets in theories with $N_{S}$ superPoincaré supercharges, its origin lies in the fact that in superspace, the four-point function depends on $4 \times \frac{N_{S}}{2}=2 N_{S}$ independent Grassmann variables. As $2 N_{S}$ is also the total number of fermionic generators

\footnotetext{
${ }^{9}$ We note that, should the reader prefer using another, more canonical, convention, our results for the superconformal blocks can be converted into any other convention for the projectors by rescaling all the coefficients, $f_{\tilde{\Delta}, \tilde{\ell}}^{J}$, according to $f_{\tilde{\Delta}, \tilde{\ell}}^{J} \rightarrow f_{\tilde{\Delta}, \tilde{\ell}}^{J} / P_{J}^{J_{1}\left(-J_{1}\right) J_{3}\left(-J_{3}\right)}$.
} 
of superconformal algebra, $Q$ and $S$, there exists a frame in which all Grassmann variables can be set to zero.

Vice versa this means that one can always find a superconformal completion from the four-point function of the superconformal primaries and restore the full Grassmann variable dependence. This completion should be well behaved as long as we keep the spacetime coordinates apart. It turns out that the superconformal transformation of the coordinates are singular, and for the four-point function to be well defined, these singularities needs to be cured, leading to the so-called Ward identity.

Let us shortly recall the linearised superconformal transformations, $\delta$, with $N_{S}=$ $8[32] .{ }^{10}$ In a frame parameterised by a pair of conformal coordinates, $(\chi(z), \bar{\chi}(\bar{z}))$ - see appendix B for details - these transformations induce the following pole:

$$
\delta_{Q} w=\varepsilon \delta_{Q} \chi \sim \frac{1}{w-\chi}, \quad \chi=\frac{z}{1-z}
$$

where only the pole was kept for simplicity. We refer to the original work for more details.

A transformation of the full four-point function will therefore become singular as the auxiliary invariant, $w$, approaches the conformal coordinate, $\chi$. It is then clear that physical quantities should be free of such a singularity, which can be attained by demanding that

$$
\left.\left(\left(\partial_{\chi}+\varepsilon \partial_{w}\right)\left\langle\phi_{1}\left(x_{1}, Y_{1}\right) \phi_{2}\left(x_{2}, Y_{2}\right) \phi_{3}\left(x_{3}, Y_{3}\right) \phi_{4}\left(x_{4}, Y_{4}\right)\right\rangle\right)\right|_{\chi=w}=0 .
$$

A similar Ward identity can also be obtained for the other variable, $\bar{\chi}(\bar{z})$ by exchanging $\chi \leftrightarrow \bar{\chi}$. A convenient choice of convention for the prefactor reduces it to a particularly appealing form:

$$
\left.\left(\left(\partial_{\chi}+\varepsilon \partial_{w}\right) F(u, v ; w)\right)\right|_{\chi=w}=0, \quad a_{1}=J_{1}+J_{2}, a_{2}=J_{34}
$$

In the case of arbitrary $a_{1}, a_{2}$ however, one must take particular care of the prefactors $K_{4}, K_{4}^{R}$. Upon defining the variable $\alpha=(1+w) / w$, the superconformal Ward identity takes the form

$$
\left.\left(-z \varepsilon\left(\left(a_{1}-J_{1}-J_{2}\right)+z\left(a_{2}-J_{34}\right)\right)+(z-1)\left(z^{2} \partial_{z}-\varepsilon \partial_{\alpha}\right)\right) F(z, \bar{z}, \alpha)\right|_{\alpha=\frac{1}{z}}=0 .
$$

One can then plug in the expression of the superconformal blocks and their bosonic contributions to get explicit constraints on the coefficients $f_{\tilde{\Delta} . \tilde{\ell}}^{J}$. After the dust settles, the resulting identity depends only on $z$. As described in appendix B the factor containing $a_{1}, a_{2}$ actually cancels against factors contained in the harmonics. As a result all $a_{1}, a_{2}$ dependence of the above Ward identity is merely a relic from the choice of $K_{4}^{R}$ and drops out in actual computations. Furthermore the resulting Ward identity applied on a specific superconformal block will factor out all contributions of the form $\left(J_{1}+J_{2}\right)$, and all the external data will boils down to the specific combinations $J_{12}, J_{34}$.

\footnotetext{
${ }^{10}$ We would like to warn the reader than in [32], the use of $z$ and $\chi$ are exchanged. We use the present notation as it has become the standard in the recent literature.
} 


\subsection{Crossing symmetry constraints}

Finally, we close this section on the superconformal blocks by giving the crossing symmetries that they must satisfy. Until here, we have tacitly assumed that the OPE leading to the block decomposition was performed in the $s$-channel, that is to say by performing an OPE on $\phi_{1}$ with $\phi_{2}$ and on $\phi_{3}$ with $\phi_{4}$ respectively. The other two channels, $u$ and $s$, should be the same by the associativity properties of the OPE, and have been recently used in the numerical bootstrap to obtain bounds on the conformal data, see [8] and references therein for a review.

Let us begin by reviewing crossing symmetry in the case of bosonic blocks. First, in the $t$-channel, obtained by exchanging $(1 \leftrightarrow 3)$, one can see that the cross-ratios $(2.4)$ are exchanged, $u \leftrightarrow v$, and that the kinematic prefactor gets rescaled by powers of the external dimensions:

$$
K_{4} \stackrel{1 \leftrightarrow 3}{\longrightarrow} u^{\frac{\Delta_{1}+\Delta_{2}}{2}} v^{-\frac{\Delta_{2}+\Delta_{3}}{2}} K_{4} .
$$

In the case of coincident external bosonic operators with the same conformal dimension, $\Delta_{\phi}$, crossing symmetry leads to the well-known sum rule function $\mathcal{F}_{\Delta, \ell}(u, v)=v^{\Delta_{\phi}} g_{\Delta, \ell}(u, v)-$ $u^{\Delta_{\phi}} g_{\Delta, \ell}(v, u)$, omnipresent in bootstrap applications.

Considering the exchange $1 \leftrightarrow 2$ is also important, as it relates to the $u$-channel and can lead to important constraints. In that case, the cross-ratios change according to $(u, v) \leftrightarrow(u / v, 1 / v)$, and it can be shown that the bosonic blocks themselves satisfy the relation $[3,41]$,

$$
g^{\Delta_{12}, \Delta_{34}}(u, v)=(-1)^{\ell} v^{-\frac{\Delta_{34}}{2}} g_{\Delta, \ell}^{-\Delta_{12}, \Delta_{34}}(u / v, 1 / v),
$$

which in the coincident, non-supersymmetric case acts as a selection rule for the allowed operators, where only multiplets with even $\ell$ may be exchanged.

While we have used methods involving explicit $\mathrm{SU}(2)_{R}$ indices and auxiliary variables, $Y^{\alpha}$, throughout this work we will focus here on crossing symmetry constraints involving auxiliary variables. This has the distinct advantage of making the expressions simpler, with all the difficulty hidden in the details of the $\mathrm{SU}(2)_{R}$ harmonics. The other case leads to equivalent results, but involves so-called "Fierz", or flavour matrices [54]. To simplify expressions, we set without loss of generality the coefficients $a_{1}=J_{1}+J_{2}, a_{2}=J_{34}$, and take an ordering such that $J_{1} \leq J_{2} \leq J_{3} \leq J_{4}$. Of course, as the full four-point function does not depend on $a_{1}, a_{2}$, the choice of convention does not matter in the end.

Now, under exchange $(1 \rightarrow 3)$, the $\mathrm{SU}(2)_{R}$ cross-ratio is inverted, $w \rightarrow 1 / w$. Crossing symmetry between $s$ - and $t$-channels then imposes the relation

$$
v^{\frac{\Delta_{2}+\Delta_{3}}{2}} \mathcal{G}_{\chi}(u, v ; w)=(-1)^{J_{1}+J_{2}+J_{34}} w^{-\left(J_{1}+J_{2}\right)}(1+w)^{J_{1}-J_{3}} u^{\frac{\Delta_{1}+\Delta_{2}}{2}} \mathcal{G}_{\chi}\left(v, u ; \frac{1}{w}\right),
$$

The simplest strategy to obtain the crossing relations satisfied by the superconformal blocks, $\mathcal{G}_{\chi}(u, v ; w)$, is to remember that the harmonics are polynomials in $w^{-1}$ of degree $J_{1}+J_{2}$, and therefore so are the blocks. Matching both sides of (3.24) for given superconformal multiplet order by order in $w^{-1}$, one obtains a certain number of relations and supersymmetric versions of $\mathcal{F}_{\Delta, \ell}$ can be defined in a form suitable for the bootstrap. For coincident R-charges, $J_{i}=J$, there are $J+1$ such relations, and one can use the Ward 
identity to find $J$ independent crossing equations [26]. We note that in the bootstrap, using dependent crossing equations has been known to lead to numerical instabilities, see e.g. [55].

Let us finish by checking the kind of constraints one obtains when considering the exchange $(1 \leftrightarrow 2)$. The $\mathrm{SU}(2)_{R}$ cross ratio changing according to $w \rightarrow-w /(1+w)$, and using the standard Kummer relations for hypergeometric functions, one obtains

$$
\mathcal{P}_{J}^{-J_{12}, J_{34}}\left(\frac{-w}{1+w}\right)=(-1)^{J}(1+w)^{2 J_{34}} \mathcal{P}_{J}^{J_{12}, J_{34}}(w) .
$$

Together with the transformations of $K_{4}, K_{4}^{R}$, and the bosonic blocks under the same exchange (3.23), this gives additional constraints on the blocks. For instance, in the case where $J_{i j}=0$, this leads to the selection rule

$$
\ell+J_{R} \in 2 \mathbb{Z}, \quad \text { if } J_{12}=0=J_{34} .
$$

Notice that the same constraints also apply to descendants, $\tilde{\ell}+J \in 2 \mathbb{Z}$. As we will see when solving the constraints discussed in this section, the superconformal block knows about it, and the coefficient of a would-be violating descendant precisely vanishes in that case.

\section{Superconformal blocks of mixed $\mathcal{D}$-type four-point functions}

We are now ready to solve the constraints derived in the last section, and find the explicit form of the superconformal blocks associated to each of the superconformal multiplets. We consider blocks appearing in the four-point function of four scalar superconformal primaries, $\phi_{i}$, belonging to short superconformal multiplets, $\mathcal{D}\left[J_{i}\right]$. Finding an explicit form for the superconformal block involves determining the coefficients, $f_{\tilde{\Delta}, \tilde{\ell}}^{J}$, of the bosonic conformal blocks in the expansion (2.25). These are determined by considering the type (II) Casimir equation (3.17) and the superconformal Ward identity (3.21). As we will find below, while the type (II) constraint fixes the coefficients, $f_{\tilde{\Delta}, \tilde{\ell}}^{J}$, for all of the contributing $\mathcal{D}$-type blocks, it is in general not strong enough to find unique solutions for all types of superconformal multiplets. On the other hand, the Ward identity will be able to fix uniquely — or forbid - all possible superconformal blocks.

In the general case, where we consider the superconformal blocks associated to e.g. long multiplets, the expressions for the coefficients are given as ungainly rational functions of the various conformal data. For the convenience of the reader who might want to use them, they have been included in the supplementary material, and they are directed there for the complete list of superconformal blocks. Here, we write the results for some of the edge cases, such as the superconformal blocks for the short multiplets, or some of the more manageable coefficients of the long superconformal multiplets.

Solving the constraints. As mentioned already, the constraints follow from applying differential operators to the bosonic blocks. With the exception of $\mathcal{D}_{\text {bos }}$, the bosonic blocks are not eigenfunctions of these operators, and this must be dealt with. To do so, we use 
introduce a decomposition in terms of Jack polynomials, $P_{\lambda_{1}, \lambda_{2}}^{(\varepsilon)}$, of the bosonic blocks [4, 41],

$$
g_{\Delta, \ell}^{\Delta_{12}, \Delta_{34}}(z, \bar{z})=\sum_{m, n \geq 0} r_{m, n}\left(\Delta_{12}, \Delta_{34}, \Delta, \ell\right) P_{\frac{1}{2}(\Delta+\ell)+m, \frac{1}{2}(\Delta-\ell)+n}^{(\varepsilon)}(z, \bar{z}) .
$$

The coefficients, $r_{m, n}$, of the infinite series depend solely on the conformal data, and can be determined recursively by application of the bosonic Casimir operator (3.2),

$$
\begin{aligned}
r_{m, n}\left(\Delta_{12}, \Delta_{34}, \Delta, \ell\right)= & \left(\frac{1}{2}\left(\Delta+\ell-\Delta_{12}\right)\right)_{m}\left(\frac{1}{2}\left(\Delta+\ell+\Delta_{34}\right)\right)_{m} \\
& \times\left(\frac{1}{2}\left(\Delta-\ell-\Delta_{12}\right)-\varepsilon\right)_{n}\left(\frac{1}{2}\left(\Delta-\ell+\Delta_{34}\right)-\varepsilon\right)_{n} \widehat{r}_{m, n}^{\Delta, \ell},
\end{aligned}
$$

where $(a)_{n}$ denotes the Pochhammer symbol. The quantity $\widehat{r}_{m, n}^{\Delta, \ell}$ can be evaluated recursively via the relation

$$
\begin{aligned}
(m(m+\Delta+\ell-1) & +n(n+\Delta-\ell-2 \varepsilon-1)) \widehat{r}_{m, n}^{\Delta, \ell} \\
& =\frac{\ell+m-n-1+2 \varepsilon}{\ell+m-n-1+\varepsilon} \widehat{r}_{m-1, n}^{\Delta, \ell}+\frac{\ell+m-n+1}{\ell+m-n+1+\varepsilon} \widehat{r}_{m, n-1}^{\Delta, \ell},
\end{aligned}
$$

together with the initial condition $r_{0,0}=1$. We note that a closed form of this recursion relation is known in terms of a generalised hypergeometric function, ${ }_{4} F_{3}$, but it will not be necessary here [4].

Using the properties of the Jack polynomials collected in appendix C, one can infer the action of $\mathcal{D}_{\text {SUSY }}$ as

$$
\begin{aligned}
\mathcal{D}_{\mathrm{SUSY}} P_{\lambda_{1}, \lambda_{2}}^{(\varepsilon)}= & 4\left(\lambda_{1}+\lambda_{2}\right) P_{\lambda_{1}, \lambda_{2}}^{(\varepsilon)}-2 \frac{\left(\Delta_{34}+2 \lambda_{1}\right)\left(\lambda_{1}-\lambda_{2}+2 \varepsilon\right)}{\lambda_{1}-\lambda_{2}+\varepsilon} P_{\lambda_{1}+1, \lambda_{2}}^{(\varepsilon)} \\
& -2 \frac{\left(\lambda_{1}-\lambda_{2}\right)\left(\Delta_{34}+2 \lambda_{2}-2 \varepsilon\right)}{\lambda_{1}-\lambda_{2}+\varepsilon} P_{\lambda_{1}, \lambda_{2}+1}^{(\varepsilon)} .
\end{aligned}
$$

This formula has no explicit dependence on $z, \bar{z}$ and makes the Jack polynomials very good candidates to deal with the full superconformal Casimir operator. Indeed, the action of the Casimir operator on a conformal block gives a linear combination of Jack polynomials. As there is no explicit dependence on spacetime coordinates, one can use the fact that they form an orthogonal basis of symmetric polynomials to solve the constraints order by order. This method has proven very efficient to find the form of the superconformal blocks of momentum map operators in this context, see e.g. [27, 34].

In the case of the Ward identity, we have not determined a way to write the differential operator (3.21) on a Jack polynomial in a closed form, i.e. in such a way that it involves only a linear combination of Jack polynomials without explicit dependence on the coordinates. In [26], the Ward identity associated to external momentum maps operators, $\mathcal{D}[1]$, was solved using various inversion formulae [32]. While these formulae can be generalised for higher external spins, we find the following procedure more convenient: use the properties of Jack polynomials (see appendix C) and of hypergeometric functions to reduce the Ward identity to a manageable, albeit long, form; perform an expansion in radial coordinates, in the same spirit of [49]; and finally solve the constraints order by order. 
In all cases, the above procedures lead to a linear set of equations involving the superconformal block coefficients of the form

$$
\sum_{J, \tilde{\Delta}, \tilde{\ell}} \alpha_{\tilde{\Delta}, \tilde{\ell}}^{J} f_{\tilde{\Delta}, \tilde{\ell}}^{J}=0
$$

where the sum is taken over all possible non-supersymmetric conformal primaries inside the multiplet under consideration, and $\alpha_{\tilde{\Delta}, \tilde{\ell}}^{J}$ is a function of the superconformal data, possibly vanishing. It is then clear that one of two possibilities can occur: either all the coefficients are proportional to that of the superconformal primary - that we safely set to one - or they all vanish and the multiplet does not appear in the block expansion. While tedious, determining the set of constraints from the above procedure can be implemented algorithmically in a straightforward manner, up to a given order. If a solution is found to be valid up to some threshold, one can then easily check that it is satisfied to all orders by reinjecting it in the original equation and using the recursion relations (4.2) and (4.3).

Let us now outline the result of this procedure and comment on some features for all types of superconformal multiplets, going from simplest to most complex (i.e. largest size of the) multiplets.

$\mathcal{D}$-type multiplets. Let us apply both methods to the simplest class of multiplets, type $\mathcal{D}\left[J_{R}\right]$. In this case, the terms in the expansion (2.25) of the superconformal block are particularly simple, making them a good stepping stone to the other, more complicated, multiplets. Indeed, their primaries can only be scalars, and there are only three possible states in the $\ell$-symmetric representation of the Lorentz group:

$$
(\tilde{\Delta}, \tilde{\ell}, J) \in\left\{\left(2 \varepsilon J_{R}, 0, J_{R}\right),\left(2 \varepsilon J_{R}+1,1, J_{R}-1\right),\left(2 \varepsilon J_{R}+2,0, J_{R}-2\right)\right\} .
$$

The first entry of the list corresponds to the superconformal primary. Plugging in this spectrum in both the Casimir and Ward identity equations, all constraints can be uniquely solved and the following values for the coefficients of the superconformal blocks are found:

$$
\begin{aligned}
& f_{2 \epsilon J_{R}+1,1}^{J_{R}-1}=\frac{\epsilon\left(J_{R}+J_{12}\right)\left(J_{R}+J_{34}\right)}{J_{R}\left(2 \epsilon J_{R}+1\right)} \\
& f_{2 \epsilon J_{R}+2,0}^{J_{R}-2}=\frac{\epsilon^{2}\left(J_{R}+J_{12}\right)\left(J_{R}+J_{34}\right)\left(J_{R}+J_{12}-1\right)\left(J_{R}+J_{34}-1\right)}{2\left(J_{R}-1\right)\left(2 J_{R}-1\right)\left(2 \epsilon J_{R}+1\right)\left(2 \epsilon J_{R}-\epsilon+1\right)} .
\end{aligned}
$$

These results generalise those found in four dimensions, [23, 33], and for external momentum maps $[26,27]$.

Note that the structure of the blocks naturally encodes a lot of information about the representation theory of the conformal group. For instance, the trivial multiplet, $\mathcal{D}[0]$, corresponds to the identity operator, which has no superpartners and can only appear if $J_{12}=0=J_{34}$. In that case there is only one coefficient in the expansion of the superconformal block, that of the lone primary, while the others vanish. Another example is that of the momentum map, $\mathcal{D}[1]$, containing a null-state at level $J_{R}-2$ corresponding to the conservation of the flavour current, $\partial_{\mu} \mathcal{J}^{\mu}=0$. When it is allowed in the OPE, the block "knows" that there is no corresponding state at that level, and its coefficient vanishes. The 
same kind of remarks will also apply with different conserved quantities in other types of multiplets.

We are left with one constraint still unchecked, that of the type (I) Casimir equation (3.15). For $\mathcal{D}$-type multiplets, a quick inspection of all possibilities reveals that the only state satisfying the constraint is the primary. This means that any such multiplet with R-charge $J_{R} \in \mathcal{J}$ is allowed as an exchanged operator in the decomposition.

$\mathcal{B}$-type multiplets. For $\mathcal{B}$-type multiplets, the states that can possibly appear in the superconformal blocks are

$$
\begin{aligned}
(\tilde{\Delta}, \tilde{\ell}, J) \in\{ & \left(\Delta, \ell, J_{R}\right), \\
& \left(\Delta+1, \ell \pm 1, J_{R}-1\right),\left(\Delta+1, \ell+1, J_{R}\right),\left(\Delta+1, \ell+1, J_{R}+1\right), \\
& \left(\Delta+2, \ell, J_{R}-2\right),\left(\Delta+2, \ell, J_{R}-1\right),\left(\Delta+2, \ell, J_{R}\right),\left(\Delta+2, \ell+2, J_{R}\right), \\
& \left.\left(\Delta+3, \ell+1, J_{R}-1\right)\right\} .
\end{aligned}
$$

In this case, the type (I) Casimir equation is never satisfied by the primary, which excludes $\mathcal{B}\left[\ell, J_{R}=J_{\max }\right]$ to appear in the block decomposition. The state with $\left(J_{R}+1\right)$ is however the only one satisfying the constraints, which mean that the biggest R-charge these multiplets can achieve is $J_{R}=J_{\max }-1$.

The type (II) Casimir equation is no longer strong enough to fix all of the coefficients due to an increased number of possible states. The linear system (4.5) is indeed underdetermined and one cannot find a unique solution. Previous works [27, 34] looked at cases where the type (II) Casimir equation was always enough to get a unique solution, but this fails when considering more involved cases. This can be disheartening, but we have a second set of constraints: the Ward identity. Such an approach does not suffer from this drawback and is in fact able to find a unique solution for any multiplet. One can check that the underdetermined system of the Casimir is satisfied by that solution. Due to the lengthy expression of the results, we have tabulated them in appendix (D).

Note that in section 3.3, we have seen that if all external scalars have the same Rcharge, the states in equation (4.9) must satisfy $J+\tilde{\ell} \in 2 \mathbb{Z}$. There are two states violating this condition: $\left(\Delta, \ell+1, J_{R}\right)$ and $\left(\Delta, \ell, J_{R}-1\right)$. As one can see from equation (D.2), the superconformal blocks know about this selection rule and their coefficients automatically vanish should the primary be allowed.

$\mathcal{C}$ - and $\mathcal{A}$-type multiplets. As has been already mentioned in the introduction, the $\mathcal{C}$-type is special to six dimensions due to the presence of tensor multiplets. In that case, both the Casimir equation and the Ward identity lead to solutions only if all coefficients, including that of the primary, vanish. This indicates that the superconformal blocks for these multiplets cannot appear in the block decomposition of the four-point function of any half-BPS states. Again, this generalises the results of momentum map operators to higher external R-charges [26, 43].

We find a similar behaviour in the case of type $\mathcal{A}$. While the Casimir equation again fails to fix all coefficients uniquely, the Ward identity ultimately shows that the supercon- 


\begin{tabular}{|c|c|}
\hline$J_{R}-2$ & $(\Delta+2, \ell)$ \\
\hline$J_{R}-1$ & $(\Delta+1, \ell \pm 1),(\Delta+2, \ell),(\Delta+3, \ell \pm 1)$ \\
\hline$J_{R}$ & $(\Delta, \ell),(\Delta+1, \ell \pm 1),(\Delta+2, \ell \pm 2),(\Delta+2, \ell),(\Delta+3, \ell \pm 1),(\Delta+4, \ell)$ \\
\hline$J_{R}+1$ & $(\Delta+1, \ell \pm 1),(\Delta+2, \ell),(\Delta+3, \ell \pm 1)$ \\
\hline$J_{R}+2$ & $(\Delta+2, \ell)$ \\
\hline
\end{tabular}

Table 1. $\ell$-symmetric states potentially appearing in a long superconformal multiplet.

formal block is trivial. From this, we conclude that both $\mathcal{C}$ - and $\mathcal{A}$-type multiplets do not appear in the block expansion.

Long superconformal multiplets. Finally, we arrive at a discussion of the long multiplets. Such multiplets contain a total of twenty-one different states in a traceless-symmetric representation of the Poincaré group, which we summarise in table 1. An analysis of these states against the type (I) Casimir equation reveals that none of them can satisfy it, except for the lone state with R-charge $J=J_{R}+2$, which implies that long multiplets can only appear in the decomposition if they have $J_{R} \leq J_{\max }-2$, as was claimed in equation (2.25).

The type (II) Casimir equation is again not able to find a unique solution for the full superconformal block, while the Ward identity fixes it completely. The procedure for long multiplets remains completely the same as for short multiplets, albeit for much longer expressions due to the fact that conformal dimensions are not fixed by unitarity. The results, for all the allowed coefficients, $f_{\tilde{\Delta}, \tilde{\ell}}^{J}$, quickly become long and unwieldy, and as such we have included an exhaustive list of the coefficients in the supplementary material. We however have written a selected list of the simplest coefficients in appendix D.

Interestingly, we find that - at least some of - the coefficients are related to one another by linear shifts of the conformal data. For instance, if two states $(\tilde{\Delta}, \tilde{\ell}, J \pm j)$ both appear in the spectrum for a given $j$, they are related to one another by a linear transformation of $J_{R}$ in their respective expression. By looking at level one for instance, equation (D.3), it is easy to convince oneself that

$$
f_{\Delta+1, \ell \pm 1}^{J_{R}-1}=\left.f_{\Delta+1, \ell \pm 1}^{J_{R}+1}\right|_{J_{R} \rightarrow-\left(J_{R}+1\right)} .
$$

This behaviour between fixed conformal parameter and R-charge generalises to higher levels, sometimes involving shifts of $\ell$ as well

$$
\begin{aligned}
& f_{\Delta+2, \ell}^{J_{R}-2}=\left.f_{\Delta+2, \ell}^{J_{R}+2}\right|_{J_{R} \rightarrow-\left(J_{R}+1\right)}, \\
& f_{\Delta+2, \ell}^{J_{R}-1}=\left.f_{\Delta+2, \ell}^{J_{R}+1}\right|_{J_{R} \rightarrow-\left(J_{R}+1\right), \ell \rightarrow-(\ell+2 \varepsilon)}, \\
& f_{\Delta+3, \ell}^{J_{R}-1}=\left.f_{\Delta+2, \ell}^{J_{R}+1}\right|_{J_{R} \rightarrow-\left(J_{R}+1\right)} .
\end{aligned}
$$

Similar relations exist for fixed R-charge and shifted Poincaré representation:

$$
f_{\tilde{\Delta}, \ell+1}^{J}=\left.\left(f_{\tilde{\Delta}, \ell-1}^{J}\right)\right|_{\ell \rightarrow-(\ell+2 \varepsilon)} .
$$


This relation is valid for all fixed $(\tilde{\Delta}, J)$ with $\ell \pm 1$ appearing in table 1 . Those linear transformations can be traced back to the four accidental $\mathbb{Z}_{2}$ symmetries of the superconformal Casimir (3.7),

$$
\begin{aligned}
(\Delta, \ell) & \longleftrightarrow(-(\ell+1),-(\Delta+1)) \\
\Delta & \longleftrightarrow-(\Delta+D-4) \\
\ell & \longleftrightarrow-(\ell+(D-2)) \\
J_{R} & \longleftrightarrow-\left(J_{R}+1\right)
\end{aligned}
$$

While we were unable to find relations between states that are not shifted from the data of the primary by the same increment, e.g. finding a transformation between $f_{\tilde{\Delta}, \tilde{\ell}}^{J_{R}}$ and $f_{\tilde{\Delta}, \tilde{\ell}}^{J_{R}-1}$ or the more complicated coefficients, they hint at a perhaps more fundamental form of the superconformal blocks. It was indeed pointed out in $[34,56]$ that in four dimensions, the superconformal Casimir equation for coincident external primaries in $\mathcal{N}$ extended supersymmetry is satisfied by $\mathcal{G}=u^{-\mathcal{N} / 2} g_{\Delta+\mathcal{N}, \ell}^{\Delta_{12}=\Delta_{34}=\mathcal{N}}(u, v)$, although it is not obvious that such that such an expression can be decomposed back into bosonic blocks with $\Delta_{i j}=0$. Our findings hint at a generalisation of this expression to arbitrary dimensions.

Finding such a fundamental form of the superconformal blocks could potentially enable to find faster algorithms for the superconformal bootstrap, leading to stricter bounds, as the form above is considerably simpler than that of equations (2.15) or (2.24) with the coefficients as determined herein. We leave further analysis in this direction for future work.

We close this section by commenting on the blocks of long multiplets in the limit when the external fields have the same R-charge. While this certainly makes the expressions simpler, the coefficient $f_{\Delta+2, \ell}^{J_{R}}$ remains particularly long. A simplification of note is that there are six coefficients that vanish identically in that limit. As in the case of $\mathcal{B}$-type multiplets, this is traced back to the condition (3.26) that these states must satisfy $\tilde{\ell}+J=0 \bmod 2$.

\section{Conclusions}

In this work we provide explicit expressions for the superconformal blocks associated to each superconformal multiplet that appears inside of the four-point function of scalars contained inside of $\mathcal{D}$-type multiplets for theories with eight supercharges in arbitrary dimensions $2<d \leq 6$. These results generalise previous work [21-27] on the topic to arbitrary R-symmetry representations and to mixed correlators of primaries from different $\mathcal{D}$-type multiplets. In particular, we find that also for $R$-spin greater than one, only three out of the five types of superconformal multiplets are allowed in the superconformal block expansion of the four-point function of a priori distinct superconformal primaries of halfBPS multiplets.

The Ward identity was, as expected, powerful enough to find a unique solution for all the blocks, where such a computation was feasible to do, or else to exclude them from the expansion altogether. On the other hand the Casimir equation was not able to fix the form of the superconformal blocks completely, but only impose a set of relations between the involved coefficients. One could think that other constraints might come from the richer structure of the projectors that was not present in previous works, but even for 
coincident external primaries, where the form of the type (II) Casimir equation is unique, we were not able to determine all coefficients. It may be the case that in order to find a unique solution, one must resort to also studying the Casimir equations that arise when considering correlation functions that do not involve states carrying the extremal weights of the $\mathrm{SU}(2)_{R}$ representations, i.e., to go beyond the conditions discussed in (3.9). One would indeed expect that the Casimir equation contains the same amount of information about the blocks as the Ward identity.

These solutions follow an intriguing web of transformation relating them, leading one to think that there might be a more fundamental structure of the superconformal blocks. It would be interesting to see if this is related to - or at least could help understand - the algebraic structure of protected operators discovered in four-dimensional $\mathcal{N}=2 \mathrm{SCFT}$ s and its generalisations [28]. Finding a more compact form for the blocks could also potentially improve the rapidity of current numerical applications to the superconformal bootstrap.

The most obvious application of our results is indeed to the numerical bootstrap. So far, most of the literature has focused on external primaries associated to half-BPS multiplets containing flavour currents or the energy-momentum tensor, depending on the number of supercharges. Our results enable a line of research going beyond this and potentially allows for an extension of the known set of bounds. Furthermore we have not made any special kind of assumption for the nature of the external primaries except that they are of type $\mathcal{D}\left[J_{i}\right]$. The blocks also enable a study of mixed correlators, which have led to strong bounds on conformal data and insights on possibly minimal supersymmetric CFTs $[24,30,52,57-62]$.

Due to their independence of the dimension of spacetime, our results are also predisposed to studies on six-dimensional SCFTs, a field that has remained quite unexplored compared to lower dimensions (see $[26,63]$ for the works that initiated the program). Furthermore a conjecturally complete classification of these theories has been achieved using string theory [64-66] (see [67] for a review). The relationship between conformal field theory and the compactification geometry seems to indicate that the conformal data might be tightly controlled by geometric invariants. As an example, the central charge of these theories is related to intersection numbers of the underlying elliptic fibration via anomaly polynomial relations. A natural question is then to ask whether the bootstrap can shed some light on the nature of these invariants; this topic is the subject of work that will appear in the near future [68].

\section{Acknowledgments}

We thank F. Apruzzi, M. Gillioz, and M. Wiesner for helpful discussions. F.B. is especially grateful to Riccardo Rattazzi and the theory group at EPFL for hospitality and stimulating discussions in the infancy of this project. C.L. would like to thank the 2019 Pollica Summer Workshop, supported in part by the Simons Collaboration on the Non-perturbative Bootstrap and in part by the INFN, for a stimulating environment during important stages of this work. The work of F.B. and M.F. is supported through the grants SEV-20160597, FPA2015-65480-P and PGC2018-095976-B-C21 from MCIU/AEI/FEDER, UE. C.L. is supported by NSF CAREER grant PHY-1756996. 


\section{A The superconformal group}

We are interested in superconformal field theories whose bosonic subalgebra is $\mathfrak{s o}(1, d+$ 1) $\times \mathfrak{s u}(2)_{R}$. We follow the work of [42] and impose a reality constraint on the generators compatible with unitarity in Lorentzian signature. Namely, we choose a basis of generators satisfying the constraints

$$
D^{\dagger}=-D, \quad M_{\mu \nu}^{\dagger}=M_{\mu \nu}, \quad P_{\mu}^{\dagger}=K_{\mu}, \quad R_{i}^{\dagger}=R_{i},
$$

where: $\left(P_{\mu}, M_{\mu \nu}\right)$ are the usual generators of the Poincaré group; $D$ and $K$ are the generators of dilatations and special conformal transformations respectively; and $R_{i}$ the generators of the R-symmetry group. Chosen so, the generators satisfy the usual Euclidean conformal algebra

$$
\begin{array}{rlrl}
{\left[M_{\mu \nu}, M_{\rho \sigma}\right]} & =-i\left(\delta_{\mu \sigma} M_{\nu \rho}+\delta_{\nu \rho} M_{\mu \sigma}-\delta_{\mu \rho} M_{\nu \sigma}-\delta_{\nu \sigma} M_{\mu \rho}\right) \\
{\left[M_{\mu \nu}, P_{\rho}\right]} & =-2 i P_{[\mu} \delta_{\nu] \rho}, & {\left[M_{\mu \nu}, K_{\rho}\right]} & =-2 i K_{[\mu} \delta_{\nu] \rho}, \\
{\left[D, P_{\mu}\right]} & =-i P_{\mu}, & {\left[D, K_{\mu}\right]} & =i K_{\mu}, \\
{\left[P_{\mu}, K_{\nu}\right]} & =-2 i\left(\delta_{\mu \nu} D+M_{\mu \nu}\right), & {\left[R_{i}, R_{j}\right]} & =i \varepsilon_{i j k} R_{k} .
\end{array}
$$

In addition to the bosonic charges, there are two sets of fermionic generators, $Q_{\alpha A}, S^{\alpha A}$. They satisfy the conjugation rule, $S^{\alpha A}=\left(Q_{\alpha A}\right)^{\dagger}$. The anti-commutation relations satisfied by these fermionic generators can be obtained by considering the six-dimensional SUSY algebra and reducing to lower dimension. It was shown in [27] that to be closed under Jacobi identities, they must take the form:

$$
\begin{aligned}
& \left\{Q_{\alpha A}, Q_{\beta B}\right\}=\varepsilon_{\alpha \beta} \Gamma_{A B}^{\mu} P_{\mu}, \quad\left\{S^{\alpha A}, S^{\beta B}\right\}=\varepsilon_{\alpha \beta} \widetilde{\Gamma}_{\mu}^{A B} K_{\mu}, \\
& \left\{S^{\alpha A}, Q_{\beta B}\right\}=i \delta_{\beta}^{\alpha} \delta_{B}^{A} D-(d-2) \delta_{B}^{A} R_{\beta}^{\alpha}+\delta_{\beta}^{\alpha}\left(m_{\mu \nu}\right)_{B}^{A} M_{i j},
\end{aligned}
$$

with $\left(m_{\mu \nu}\right)=-\frac{i}{2} \widetilde{\Gamma}_{[\mu} \Gamma_{\nu]}$ and $R_{\beta}^{\alpha}=R_{i}\left(\sigma_{i}\right)_{\beta}^{\alpha}$, where $\sigma_{i}$ are the Pauli matrices. As we are considering theories with eight supercharges, $N_{S}=8$, one has $\delta_{\alpha}^{\alpha} \delta_{A}^{A}=8$. This can be generalised to a higher or lower number of supercharges by changing $\varepsilon_{\alpha \beta} \rightarrow \Omega_{\alpha \beta}$ to the appropriate pairing. Moreover, the precise form of $\Gamma^{\mu}, \widetilde{\Gamma}_{\mu}$ depends on the considered dimension, but it can be shown that they satisfy a Clifford-like identity, $\widetilde{\Gamma}_{(\mu} \Gamma_{\nu)}=\delta_{\mu \nu} \mathbf{1}$. This relation will be sufficient in this work, and we will not have to deal with particular explicit expressions of the spinor matrices.

The mixed fermionic-bosonic identities can be determined via Jacobi identities:

$$
\begin{aligned}
{\left[K_{\mu}, Q_{\alpha A}\right] } & =\varepsilon_{\alpha \beta} \Gamma_{A B}^{\mu} S^{\beta B}, & {\left[P_{\mu}, S^{\alpha A}\right] } & =-\varepsilon^{\alpha \beta} \widetilde{\Gamma}_{\mu}^{A B} Q_{\beta B}, \\
{\left[M_{\mu \nu}, Q_{\alpha A}\right] } & =\left(m_{\mu \nu}\right)^{B}{ }_{A} Q_{\alpha B}, & {\left[M_{\mu \nu}, S^{\alpha A}\right] } & =-\left(m_{\mu \nu}\right)_{B}^{A} S^{\alpha B}, \\
{\left[R_{i}, Q_{\alpha A}\right] } & =\frac{1}{2}\left(\sigma_{i}\right)_{\alpha}^{\beta} Q_{\beta A}, & {\left[R_{i}, S^{\alpha A}\right] } & =-\frac{1}{2}\left(\sigma_{i}\right)_{\beta}^{\alpha} S^{\beta A},
\end{aligned}
$$


The quadratic Casimir is then given by ${ }^{11}$

$$
\begin{aligned}
C^{2} & =C_{\mathrm{bos}}^{2}+C_{\mathrm{SUSY}}^{2}+C_{R}^{2}, \\
C_{\mathrm{bos}}^{2} & =\frac{1}{2} M_{\mu \nu} M^{\mu \nu}-D^{2}-P_{(\mu} K_{\nu)}, \\
C_{\mathrm{SUSY}}^{2} & =\frac{1}{2}\left[S^{\alpha A}, Q_{\alpha A}\right], \quad C_{R}^{2}=-2 \varepsilon R_{i} R^{i} .
\end{aligned}
$$

\section{B Conformal frame and $\mathrm{SU}(2)_{R}$ harmonics}

When dealing with homogeneous function such as the four-point function, it is often useful to go to a specific conformal frame, where the dependence on given coordinates is clearer. In this work, we deal with three different sets of coordinates, related to the conformal cross-ratios (2.4):

$$
u=z \bar{z}=\frac{\chi \bar{\chi}}{(1+\chi)(1+\bar{\chi})}, \quad v=(1+z)(1+\bar{z})=\frac{1}{(1+\chi)(1+\bar{\chi})} .
$$

The coordinates $u, v$ and $z, \bar{z}$ are by now standard in the literature, see e.g. [6-8] and references therein. The third, $\chi$, can be obtained by going in the following Lorentzian frame.

$$
\begin{aligned}
& x_{1}=\left(1+\frac{1}{2}(\chi+\bar{\chi}), \frac{1}{2}(\chi-\bar{\chi}), 0, \ldots, 0\right) \\
& x_{2}=(1,0, \ldots, 0), \quad x_{3}=\left(x_{4}\right)^{-1}=0 .
\end{aligned}
$$

The kinematic prefactor, $K_{4}$ in the four-point function then turns into

$$
K_{4} \rightarrow(\chi \bar{\chi})^{\frac{-\left(\Delta_{1}+\Delta_{2}\right)}{2}}((\chi+1)(\bar{\chi}+1))^{\frac{-\Delta_{34}}{2}},
$$

where $\Delta_{i j}=\Delta_{i}-\Delta_{j}$. These coordinates are quite useful, as explained in the main text, as under a supersymmetric transformation in superspace, they acquire a pole

$$
\delta \chi \sim \frac{1}{w-\chi}, \quad \delta w=\varepsilon \delta \chi .
$$

$\mathbf{S U}(\mathbf{2})_{\boldsymbol{R}}$ harmonics. We now derive the $\mathrm{SU}(2)_{R}$ harmonics, (2.22), by inserting the quadratic Casimir of $\mathrm{SU}(2)_{R}$ into the four-point function. Let us start with the four-point function of four - in general non-identical - primaries depending on the auxiliary variable, $Y^{\alpha}$ introduced in section 2.2,

$$
\left\langle\phi_{1}\left(x_{1}, Y_{1}\right) \phi_{2}\left(x_{2}, Y_{2}\right) \phi_{3}\left(x_{3}, Y_{3}\right) \phi_{4}\left(x_{4}, Y_{4}\right)\right\rangle=K_{4} K_{4}^{R} F(u, v ; w) .
$$

From $\mathrm{SU}(2)_{R}$ invariance it is clear that the four-point function may depend on the $Y_{i}$ through the $\mathrm{SU}(2)_{R}$ invariant product

$$
Y_{i j}:=Y_{i} \cdot Y_{j}=Y_{i}^{\alpha} Y_{j}^{\beta} \varepsilon_{\alpha \beta} .
$$

\footnotetext{
${ }^{11}$ In order to make the Casimir and $\left\{S^{\alpha} A, Q_{\beta B}\right\}$ dimension independent, one needs to add contributions from the transverse coordinates $d<\hat{\mu} \leq 6$. As we are only interested in traceless-symmetric representations of the Lorentz group in a given dimension, and therefore uncharged under extra R-symmetry factors in this work, we will not take these additional pieces into account. We refer to [27] for more details.
} 
Furthermore the primaries, $\phi_{i}\left(x_{i}, Y_{i}\right)$, must be homogeneous functions of degree $\left(-\Delta_{i}, 2 J_{i}\right)$, and the correlator (B.6) will depend on a single scale invariant cross ratio, $w$, satisfying a completeness relation:

$$
w=\frac{\left(Y_{1} \cdot Y_{2}\right)\left(Y_{3} \cdot Y_{4}\right)}{\left(Y_{1} \cdot Y_{4}\right)\left(Y_{2} \cdot Y_{3}\right)}, \quad 1+w=\frac{\left(Y_{1} \cdot Y_{3}\right)\left(Y_{2} \cdot Y_{4}\right)}{\left(Y_{1} \cdot Y_{4}\right)\left(Y_{2} \cdot Y_{3}\right)}
$$

The homogeneity property of (B.6) allows to separate the four-point function into a prefactor, $K_{4}^{R}$, which has the correct scaling and a function of the invariant cross-ratio, $w$. Enforcing this scaling behaviour in an ansatz, $K_{4}^{R}=Y_{12}^{a_{1}} Y_{13}^{a_{2}} Y_{14}^{a_{3}} Y_{23}^{a_{4}} Y_{24}^{a_{5}} Y_{34}^{a_{6}}$, fixes four out of six parameters, leading to the most general form,

$$
\begin{aligned}
K_{4}^{R}= & \left(Y_{12}\right)^{a_{1}}\left(Y_{13}\right)^{a_{2}}\left(Y_{14}\right)^{-a_{1}-a_{2}+2 J_{1}}\left(Y_{23}\right)^{-a_{1}-a_{2}+J_{12}^{+}+J_{34}} \\
& \times\left(Y_{24}\right)^{a_{2}-J_{12}-J_{34}}\left(Y_{34}\right)^{a_{1}-J_{12}^{+}+J_{34}^{+}},
\end{aligned}
$$

where we defined $J_{i j}=J_{i}-J_{j}$ and $J_{i j}^{+}=J_{i}+J_{j}$. Notice that two parameters $a_{1}, a_{2}$ are still undetermined, and correspond to rescaling of the function $F(u, v ; w)$ (see equation (2.17)) by factors of $w$ and $(1+w)$. To better see this, it is instructive to go to a specific Rsymmetry frame, which is used in the derivation of the superconformal Ward identity. It is defined as

$$
\begin{aligned}
Y_{i} & =\left(1, y_{i}\right) \Rightarrow Y_{i j}=y_{i}-y_{j}, \\
y_{1} & =1+w, \quad y_{2}=1, \quad y_{3}=y_{4}^{-1}=0, \\
K_{4}^{R} & \rightarrow w^{a_{1}}(1+w)^{a_{2}}
\end{aligned}
$$

Therefore different choices of $a_{1}, a_{2}$ can be taken care of by absorbing $w^{a_{1}}(1+w)^{a_{2}}$ into the definition of $F(u, v ; w)$.

We will now determine the $w$ dependence of $F(u, v ; w)$ by determining the harmonic functions of $\mathrm{SU}(2)_{R}$ by deriving the differential equation associated to the quadratic Casimir $C^{2}=R_{i} R^{i}$. The logic of the derivation is well known, but by the simplicity of SU(2) allows to show an easy example that can then be applied mutatis mutandis to the superconformal Casimir in section C.

The first step of the procedure is to insert a projector

$$
\operatorname{Proj}_{J}=\sum_{m}|J, m\rangle\langle J, m|
$$

and the R-symmetry Casimir in the middle of the four-point function to obtain the contribution of single R-symmetry channel, $F_{J}(u, v ; w)$. One has then two options: let the Casimir act directly on the projector,

$$
\left\langle\phi_{1} \phi_{2} C_{2}\left(\operatorname{Proj}_{J}\right) \phi_{3} \phi_{4}\right\rangle=J(J+1) K_{4} K_{4}^{R} F_{J}(u, v ; w),
$$

or to let it act on $\phi_{1} \phi_{2}$ - or $\phi_{3} \phi_{4}$, leading to the same results — which is achieved by letting the generators act as linear differential operators,

$$
\mathcal{D}_{i} \mathcal{O}(Y)=\frac{1}{2}\left(\sigma_{i}\right)^{\alpha}{ }_{\beta} Y^{\beta} \frac{\partial \mathcal{O}}{\partial Y^{\alpha}},
$$


where $\left\{\sigma_{i}\right\}$ are the Pauli matrices. As the Casimir acts on both $\phi_{1}$ and $\phi_{2}$, the differential operator is finally:

$$
\left\langle\phi_{1} \phi_{2} C_{2}\left(\operatorname{Proj}_{J}\right) \phi_{3} \phi_{4}\right\rangle=\left(\mathcal{D}_{1}+\mathcal{D}_{2}\right)_{i}\left(\mathcal{D}_{1}+\mathcal{D}_{2}\right)^{i}\left(K_{4} K_{4}^{R} F_{J}(u, v ; w)\right)
$$

Comparing equations (B.12), (B.14) and evaluating the differential operators leads to the second order differential equation

$$
a(w) \partial_{w}^{2} F_{J}(u, v ; w)+b(w) \partial_{w} F_{J}(u, v ; w)+c(w) F_{J}(u, v ; w)=0
$$

where the coefficients are found to be

$$
\begin{aligned}
a(w)= & w^{2}(1+w), \quad b(w)=2 w(1+w)\left(a_{1}-J_{12}^{+}\right)+w^{2}\left(1+2 a_{2}-J_{12}-J_{34}\right), \\
c(w)= & -J(J+1)+\left(\frac{w^{2}}{1+w} a_{2}\left(a_{2}-\left(J_{12}+J_{34}\right)\right)+w J_{12} J_{34}+(1+w)\left(a_{1}-J_{12}^{+}\right)^{2}\right. \\
& \left.+\left(a_{1}-J_{12}^{+}\right)\left(w\left(2 a_{2}-J_{12}-J_{34}\right)-1\right)\right) .
\end{aligned}
$$

This differential equation can be recast into a more familiar form whose polynomial solution involves a hypergeometric functions: $:^{12}$

$$
F_{J}(u, v ; w)=\mathcal{P}_{J}^{J_{12}, J_{34}, J_{12}^{+}}(w) \times F(u, v) .
$$

The function, $F(u, v)$, depending solely on conformal data is then identified to the contribution of spin $J$ to the superconformal blocks in equation (2.21), and the harmonics are given by

$$
\mathcal{P}_{J}^{J_{12}, J_{34}, J_{12}^{+}}(w)=c_{J} w^{-J-\left(a_{1}-J_{12}^{+}\right)}(1+w)^{-a_{2}}{ }_{2} F_{1}\left(-\left(J+J_{12}\right),-\left(J+J_{34}\right) ;-2 J ;-w\right) .
$$

In the main text, we drop the superscript $J_{12}^{+}$due to our choice of convention. Notice that the dependence on $a_{1}, a_{2}$ drops in the full four-point function due to (B.10).

Let us close this appendix by comparing two convenient choices of conventions that have appeared in the literature:

- $a_{1}=J_{1}+J_{2}, a_{2}=J_{34}$. In this case, the prefactor takes a form reminiscent of that of the spacetime prefactor, $K_{4}$,

$$
K_{4}^{R}=\left(Y_{12}\right)^{\frac{k_{1}+k_{2}}{2}}\left(Y_{34}\right)^{\frac{k_{3}+k_{4}}{2}}\left(\frac{Y_{14}}{Y_{24}}\right)^{\frac{k_{12}}{2}}\left(\frac{Y_{13}}{Y_{14}}\right)^{\frac{k_{34}}{2}} .
$$

In that case, the superconformal Ward identity takes the simple form $\left(\partial_{\chi}+\right.$ $\left.\varepsilon \partial_{w}\right)\left.F\right|_{\chi=w}=0$. This is the choice taken in this work and in the recent literature that have computed the superconformal blocks of four momentum-map operators via the Ward identity, e.g. [23, 26].

\footnotetext{
${ }^{12}$ Notice that the differential equation is second order and therefore has another solution, also in terms of a hypergeometric function, which can in our case be disregarded as it is non-polynomial.
} 
- $a_{1}=2 E, a_{2}=0$ with $2 E=J_{1}+J_{2}+J_{3}-J_{4}$. With this choice, the hypergeometric function involved in the harmonic (B.18) can be recast - up to a constant $\tilde{c}_{J}$ - into a very compact form in terms of a Jacobi polynomial, $P_{n}^{(\alpha, \beta)}$,

$$
F(u, v ; w)=P_{J+J_{34}}^{\left(2 J_{1}-2 E, 2 J_{2}-2 E\right)}\left(1+\frac{2}{w}\right) \times F(u, v) .
$$

This is the choice used in [33], but comes at the cost of the Ward identity receiving additional terms with respect to the other convention.

\section{Casimirs and Jack polynomials}

Deriving the conformal Casimir equation (3.8) follows in spirit the same steps used in appendix B to derive $\mathrm{SU}(2)_{R}$ harmonics, replacing the R-symmetry Casimir by its superconformal counterpart, (3.8). We follow the method of [27,34] throughout this appendix.

We look for a representation of the Casimir operator on scalar functions of spacetime. In the case of the bosonic part, the differential operator can be inferred in a straightforward manner from the embedding formalism [4]. Acting on a bosonic block, the nonsupersymmetric Casimir equation is then:

$$
\mathcal{D}_{\text {bos }} g_{\Delta, \ell}^{\Delta_{12}, \Delta_{34}}(z, \bar{z})=c_{\Delta, \ell}^{\text {bos }} g_{\Delta, \ell}^{\Delta_{12}, \Delta_{34}}(z, \bar{z}),
$$

where the eigenvalue is given in equation (3.5) and the differential operator by

$$
\begin{aligned}
\mathcal{D}_{\text {bos }} & =\mathcal{D}_{z}+\mathcal{D}_{\bar{z}}+4 \varepsilon \frac{z \bar{z}}{z-\bar{z}}\left((1-z) \partial_{z}-(1-\bar{z}) \partial_{\bar{z}}\right) \\
\mathcal{D}_{z} & =2 z^{2}(1-z) \partial_{z}^{2}-\left(2+\Delta_{34}-\Delta_{12}\right) z^{2} \partial_{z}+\frac{\Delta_{12} \Delta_{34}}{2} z
\end{aligned}
$$

To obtain the differential operator associated to the part of the Casimir involving fermionic generators, one needs to derive how supercharges act on fields at a point $x$.

Using $\mathcal{O} \phi(x)=(\mathcal{O} \phi)(x)+i x^{\mu}\left[P_{\mu}, \mathcal{O}\right] \phi(x)$ and Jacobi identities, one finds that on a single scalar field,

$$
\begin{aligned}
S^{\alpha A} \phi(x) & =\left(S^{\alpha A} \phi\right)(x)-i x^{\mu} \varepsilon^{\alpha \beta} \widetilde{\Gamma}_{\mu}^{A B}\left(Q_{\beta B} \phi\right)(x), \\
Q_{\alpha A} \phi(x) & =\left(Q_{\alpha A} \phi\right)(x), \\
C_{\mathrm{SUSY}}^{2} \phi(x) & =4 \phi(x)-\left(Q_{\alpha A} S^{\alpha A} \phi\right)(x) .
\end{aligned}
$$

The factor 4 in the last equation is related to traces over both $\mathfrak{s u}(2)_{R}$ and spinor indices, giving the total number of supercharges. A relation independent of the number of supercharges can be obtained by replace this factor by $N_{S} / 2$.

We now demand that the scalar is a superconformal primary, which by definition is annihilated by any of the conformal supercharges, $S^{\alpha A} \phi=0$. The Casimir operator acting on two scalar superconformal primaries is then found to be

$$
\begin{aligned}
C_{\mathrm{SUSY}}^{2} \phi_{1}\left(x_{1}\right) \phi_{2}\left(x_{2}\right)= & 4\left(\Delta_{1}+\Delta_{2}\right) \phi_{1}\left(x_{1}\right) \phi_{2}\left(x_{2}\right) \\
& -i\left(x_{12}\right)^{\mu} \varepsilon^{\alpha \beta} \widetilde{\Gamma}_{\mu}^{A B}\left(Q_{\alpha A} \phi_{1}\right)\left(x_{1}\right)\left(Q_{\beta B} \phi_{2}\right)\left(x_{2}\right) .
\end{aligned}
$$


We see that the Casimir equation a priori looks like it will involve the correlation functions of fermionic fields. However, simplifications occur when considering short superconformal multiplets.

To see this, let us now reestablish the R-symmetry indices and focus on $\mathrm{SU}(2)_{R}$. Using standard $\mathrm{SU}(2)$ notation, consider that the scalar field is a superconformal primary of a multiplet of type $\mathcal{D}[J], \phi^{M}$. This means that it transforms in the spin- $J$ representation, with $-J \leq M \leq J$. By definition, highest and lowest weight states are annihilated by half of the supercharges [19, 20, 42],

$$
Q_{1 A} \phi^{J}=0, \quad Q_{2 A} \phi^{(-J)}=0, \quad \forall A .
$$

This leads us to consider two different classes of correlation functions, which we refer to as type (I) and (II):

$$
(\mathrm{I}):\left\langle\phi_{1}^{J_{1}}\left(x_{1}\right) \phi_{2}^{J_{2}}\left(x_{2}\right) \phi_{3}^{M_{3}}\left(x_{3}\right) \phi_{4}^{M_{4}}\left(x_{4}\right)\right\rangle, \quad \text { (II) }:\left\langle\phi_{1}^{J_{1}}\left(x_{1}\right) \phi_{2}^{M_{2}}\left(x_{2}\right) \phi_{3}^{J_{3}}\left(x_{3}\right) \phi_{4}^{M_{4}}\left(x_{4}\right)\right\rangle .
$$

For correlation functions of type (I), the second term of equation (C.5) vanishes identically and the Casimir reduces to an application of the dilatation operator. The action of $\mathcal{D}_{\text {SUSY }}$ on a superconformal block is just multiplication.

In type (II), one can use the fact that in the limit $\left|x_{4}\right| \rightarrow \infty$ conformal invariance ensures that no information is lost, and the superconformal Ward identity $\langle Q \mathcal{O}\rangle=0$ allows to relate the type (II) Casimir equation to a correlation function where supercharges are only applied on the first field, $\left\langle Q_{\alpha A} Q_{\beta B} \phi_{1}\left(x_{1}\right) \cdots \phi\right\rangle$. Using the supersymmetry algebra, the supercharges can be converted into a translation generator. Acting on the whole fourpoint function decomposed into superconformal blocks, the differential operator will also act on the prefactor, $K_{4}$, cancelling the term proportional to $\Delta_{1}+\Delta_{2}$.

In summary, we get that the contribution of the supersymmetric part of the Casimir acts on a superconformal block as the following differential operators:

$$
\begin{aligned}
(\mathrm{I}): & \mathcal{D}_{\mathrm{SUSY}}=4\left(\Delta_{1}+\Delta_{2}\right) \\
(\mathrm{II}): & \mathcal{D}_{\mathrm{SUSY}}=4 z(1-z) \partial_{z}+4 \bar{z}(1-\bar{z}) \partial_{\bar{z}}-2(z+\bar{z}) \Delta_{34} .
\end{aligned}
$$

Finally, it is easy to see that when acting with $\mathcal{C}_{R}^{2}$ directly on the projector, the associated differential operator will act simply as multiplication involving the R-charge of the block element, $J$ :

$$
\mathcal{D}_{R} \mathcal{G}_{\chi}^{J}(u, v)=-2 \varepsilon J(J+1) \mathcal{G}_{\chi}^{J}(u, v) .
$$

Jack polynomials. When attempting to solve the constraints coming from the Ward identity of the Casimir equation, it is useful to decompose the bosonic blocks in terms of Jack polynomials. We collect here a few properties needed to solve the constraints. The Jack polynomials can be defined through the Gegenbauer polynomials, $C_{n}^{(\alpha)}$,

$$
P_{\lambda_{1}, \lambda_{2}}^{(\varepsilon)}(z, \bar{z})=\frac{\left(\lambda_{1}-\lambda_{2}\right) !}{(2 \varepsilon)_{\lambda_{1}-\lambda_{2}}}(z \bar{z})^{\frac{1}{2}\left(\lambda_{1}+\lambda_{2}\right)} C_{\lambda_{1}-\lambda_{2}}^{(\varepsilon)}\left(\frac{z+\bar{z}}{2(z \bar{z})^{1 / 2}}\right)
$$


normalised such that $P_{\lambda_{1}, \lambda_{2}}^{(\varepsilon)}(1,1)=1$. They satisfy a number of relations, compiled in e.g. [32], following in part from the properties of the Gegenbauer polynomials. We note that notation for the coordinates, $z, \chi$, in [32] and here is swapped, as we follow conventions that have become common in the recent literature.

Under derivation, the Jack polynomial satisfy

$$
z \partial_{z} P_{\lambda_{1}, \lambda_{2}}^{(\varepsilon)}(z, \bar{z})=\frac{\lambda_{1}+\lambda_{2}}{2} P_{\lambda_{1}, \lambda_{2}}^{(\varepsilon)}(z, \bar{z})+\frac{z-\bar{z}}{4 z \bar{z}} \frac{\left(\lambda_{1}-\lambda_{2}\right)\left(\lambda_{1}-\lambda_{2}+2 \varepsilon\right)}{1+2 \varepsilon} P_{\lambda_{1}, \lambda_{2}+1}^{(\varepsilon+1)}(z, \bar{z}) .
$$

Useful identities involving a Jack polynomial and the two variables are

$$
\begin{aligned}
(z+\bar{z}) P_{\lambda_{1}, \lambda_{2}}^{(\varepsilon)}(z, \bar{z}) & =\frac{\lambda_{1}-\lambda_{2}+2 \varepsilon}{\lambda_{1}-\lambda_{2}+\varepsilon} P_{\lambda_{1}+1, \lambda_{2}}^{(\varepsilon)}(z, \bar{z})+\frac{\lambda_{1}-\lambda_{2}}{\lambda_{1}-\lambda_{2}+\varepsilon} P_{\lambda_{1}, \lambda_{2}+1}^{(\varepsilon)}(z, \bar{z}), \\
(z-\bar{z})^{2} P_{\lambda_{1}, \lambda_{2}}^{(\varepsilon+1)}(z, \bar{z}) & =\frac{2(1+2 \varepsilon)}{\lambda_{1}-\lambda_{2}+1+\varepsilon}\left(P_{\lambda_{1}+2, \lambda_{2}}^{(\varepsilon)}(z, \bar{z})-P_{\lambda_{1}+1, \lambda_{2}+1}^{(\varepsilon)}(z, \bar{z})\right) .
\end{aligned}
$$

Notice that the second equation involves a Jack polynomial with argument $(\varepsilon+1)$, which allows for the derivative to be rewritten with expression involving only $(\varepsilon)$,

$$
z \partial_{z} P_{\lambda_{1}, \lambda_{2}}^{(\varepsilon)}=\frac{\lambda_{1}+\lambda_{2}}{2} P_{\lambda_{1}, \lambda_{2}}^{(\varepsilon)}+\frac{\left(\lambda_{1}-\lambda_{2}\right)\left(\lambda_{1}-\lambda_{2}+2 \varepsilon\right)}{2(z \bar{z})(z-\bar{z})\left(\lambda_{1}-\lambda_{2}+\varepsilon\right)}\left(P_{\lambda_{1}+2, \lambda_{2}+1}^{(\varepsilon)}-P_{\lambda_{1}+1, \lambda_{2}+2}^{(\varepsilon)}\right) .
$$

Finally, defining the operator $\mathcal{D}^{(n)}=z^{n} \partial_{z}+\bar{z}^{n} \partial_{\bar{z}}$, one finds

$$
\begin{aligned}
& \mathcal{D}^{(1)} P_{\lambda_{1}, \lambda_{2}}^{(\varepsilon)}(z, \bar{z})=\left(\lambda_{1}+\lambda_{2}\right) P_{\lambda_{1}, \lambda_{2}}^{(\varepsilon)}(z, \bar{z}), \\
& \mathcal{D}^{(2)} P_{\lambda_{1}, \lambda_{2}}^{(\varepsilon)}(z, \bar{z})=\frac{\lambda_{1}\left(\lambda_{1}-\lambda_{2}+2 \varepsilon\right)}{\left(\lambda_{1}-\lambda_{2}+\varepsilon\right)} P_{\lambda_{1}+1, \lambda_{2}}^{(\varepsilon)}(z, \bar{z})+\frac{\left(\lambda_{2}-\varepsilon\right)\left(\lambda_{1}-\lambda_{2}\right)}{\left(\lambda_{1}-\lambda_{2}+\varepsilon\right)} P_{\lambda_{1}, \lambda_{2}+1}^{(\varepsilon)}(z, \bar{z}) .
\end{aligned}
$$

\section{List of coefficients for superconformal blocks}

We collect in this appendix the list of coefficients, $f_{\tilde{\Delta}, \tilde{\ell}}^{J}$, of the superconformal blocks appearing in the decomposition of four-point functions of four $\mathcal{D}$-type multiplets. As commented in section (4), multiplets of type $\mathcal{A}$ and $\mathcal{C}$ do not appear in the decomposition.

$\mathcal{D}$-type multiplets. The $\mathcal{D}$-type multiplets contain only three states that contribute to the superconformal blocks. Setting the coefficient of the primary to one, the other two are given by:

$$
\begin{aligned}
& f_{2 \epsilon J_{R}+1,1}^{J_{R}-1}=\frac{\epsilon\left(J_{R}+J_{12}\right)\left(J_{R}+J_{34}\right)}{J_{R}\left(2 \epsilon J_{R}+1\right)}, \\
& f_{2 \epsilon J_{R}+2,0}^{J_{R}-2}=\frac{\epsilon^{2}\left(J_{R}+J_{12}\right)\left(J_{R}+J_{34}\right)\left(J_{R}+J_{12}-1\right)\left(J_{R}+J_{34}-1\right)}{2\left(J_{R}-1\right)\left(2 J_{R}-1\right)\left(2 \epsilon J_{R}+1\right)\left(2 \epsilon J_{R}-\epsilon+1\right)} .
\end{aligned}
$$

$\mathcal{B}$-type multiplets. $\mathcal{B}$-type multiplets have fixed conformal dimension, $\Delta=2 \varepsilon J_{R}+\ell+2 \varepsilon$, and the coefficients are found to be:

$$
\begin{aligned}
f_{\Delta+1, \ell-1}^{J_{R}-1} & =\frac{\ell \varepsilon\left(J_{12}+J_{R}\right)\left(J_{34}+J_{R}\right)}{2 J_{R}\left(2 J_{R} \varepsilon+1\right)(\ell+\epsilon)}, \\
f_{\Delta+1, \ell+1}^{J_{R}-1} & =\frac{\left(J_{12}+J_{R}\right)\left(J_{34}+J_{R}\right)(l+2 \varepsilon)\left(2 J_{R} \varepsilon+\ell+2 \varepsilon\right)}{2 J_{R}\left(2 J_{R}+1\right)(\ell+\varepsilon)\left(2 J_{R} \varepsilon+\ell+\varepsilon+1\right)},
\end{aligned}
$$




$$
\begin{aligned}
f_{\Delta+1, \ell+1}^{J_{R}}= & -\frac{J_{12} J_{34}(\ell+2 \varepsilon)\left(2 J_{R} \varepsilon+\ell+2 \varepsilon\right)}{2 J_{R}\left(J_{R}+1\right)\left(J_{R} \varepsilon+\ell+\varepsilon\right)\left(J_{R} \varepsilon+\ell+\varepsilon+1\right)}, \\
f_{\Delta+1, \ell+1}^{J_{R}+1}= & \frac{\left(-J_{12}+J_{R}+1\right)\left(-J_{34}+J_{R}+1\right)(\ell+2 \epsilon)}{2\left(J_{R}+1\right)\left(2 J_{R}+1\right)(\ell+1)}, \\
f_{\Delta+2, \ell}^{J_{R}-2}= & \frac{\varepsilon\left(J_{12}+J_{R}-1\right)\left(J_{12}+J_{R}\right)\left(J_{34}+J_{R}-1\right)\left(J_{34}+J_{R}\right)\left(2 J_{R} \varepsilon+\ell+2 \varepsilon\right)}{4\left(J_{R}-1\right) J_{R}\left(2 J_{R}-1\right)\left(2 J_{R} \varepsilon+1\right)\left(2 J_{R} \varepsilon+\ell+\varepsilon+1\right)}, \\
f_{\Delta+2, \ell}^{J_{R}-1}= & -\frac{J_{12} J_{34} \varepsilon\left(J_{12}+J_{R}\right)\left(J_{34}+J_{R}\right)(\ell+2 \varepsilon)\left(2 J_{R} \varepsilon+\ell+1\right)\left(2 J_{R} \varepsilon+\ell+2 \varepsilon\right)}{4\left(J_{R}-1\right) J_{R}^{2}\left(2 J_{R} \varepsilon+1\right)\left(J_{R} \varepsilon+\ell+\varepsilon\right)\left(J_{R} \varepsilon+\ell+\varepsilon+1\right)\left(2 J_{R} \varepsilon+\ell+\varepsilon+1\right)}, \\
f_{\Delta+2, \ell}^{J_{R}}= & \frac{\varepsilon\left(J_{R}^{2}-J_{12}^{2}\right)\left(J_{R}^{2}-J_{34}^{2}\right)\left(J_{34}+J_{R}\right)(\ell+2 \varepsilon)\left(2 J_{R} \varepsilon+\ell+1\right)\left(2 J_{R} \varepsilon+\ell+2 \varepsilon\right)}{4 J_{R}^{2}\left(2 J_{R}-1\right)\left(2 J_{R} \varepsilon+1\right)(\ell+\varepsilon+1)\left(2 J_{R} \varepsilon+\ell+\varepsilon\right)\left(2 J_{R} \varepsilon+\ell+\varepsilon+1\right)} \\
f_{\Delta+2, \ell+2}^{J_{R}}= & \frac{(\ell+2 \varepsilon)(\ell+2 \varepsilon+1)}{4(\ell+1)(\ell+\varepsilon+1)} \\
& \times \frac{\left(A^{-} \varepsilon+\ell+1\right)\left(A^{+} \varepsilon+\ell+1\right)\left(B^{-} \varepsilon+\ell+1\right)\left(B^{+} \varepsilon+\ell+1\right)\left(2\left(J_{R}+1\right) \varepsilon+\ell\right)}{\left(J_{R} \varepsilon+\ell+\varepsilon+1\right)^{2}\left(2 J_{R} \varepsilon+\ell+\varepsilon+1\right)\left(2\left(J_{R}+1\right) \varepsilon+2 \ell+1\right)\left(2\left(J_{R}+1\right) \varepsilon+2 \ell+3\right)}, \\
f_{\Delta+3, \ell+1}^{J_{R}-1}= & \frac{\varepsilon\left(J_{12}+J_{R}\right)\left(J_{34}+J_{R}\right)(\ell+2 \varepsilon)\left(A^{-} \varepsilon+\ell+1\right)\left(A^{+} \varepsilon+\ell+1\right)}{8 J_{R}(l+1)\left(2 J_{R} \varepsilon+1\right)\left(J_{R} \varepsilon+l+\varepsilon+1\right)^{2}\left(2 J_{R} \varepsilon+\ell+\varepsilon+1\right)^{2}} \\
& \times \frac{\left(B^{-} \varepsilon+\ell+1\right)\left(B^{+} \varepsilon+\ell+1\right)\left(2 J_{R} \varepsilon+\ell+2\right)\left(2\left(J_{R}+1\right) \varepsilon+\ell\right)\left(2\left(J_{R}+1\right) \varepsilon+\ell+1\right)}{\left(2 J_{R} \varepsilon+\ell+\varepsilon+2\right)\left(2\left(J_{R}+1\right) \varepsilon+2 \ell+1\right)\left(2\left(J_{R}+1\right) \varepsilon+2 \ell+3\right)}, \\
&
\end{aligned}
$$

where we defined $A^{ \pm}=J_{R}+1 \pm J_{12}, B^{ \pm}=J_{R}+1 \pm J_{34}$ and set the coefficient of the primary to one.

Long superconformal multiplets. Long multiplets have unconstrained conformal dimensions above the unitarity bound, and the superconformal blocks receive contributions from 20 different states, in addition to the superconformal primary, whose coefficient we set to one by convention.

At level one, the coefficients are given by

$$
\begin{aligned}
f_{\Delta+1, \ell-1}^{J_{R}-1} & =\frac{\ell\left(J_{12}+J_{R}\right)\left(J_{34}+J_{R}\right)\left(\Delta-\ell+2 \varepsilon J_{R}\right)}{2 J_{R}\left(2 J_{R}+1\right)(\ell+\varepsilon)\left(\Delta-\ell+2+2\left(J_{R}-1\right) \varepsilon\right)} \\
f_{\Delta+1, \ell+1}^{J_{R}-1} & =\frac{\left(J_{12}+J_{R}\right)\left(J_{34}+J_{R}\right)(\ell+2 \varepsilon)\left(\Delta+2\left(J_{R}+1\right) \varepsilon+\ell\right)}{2 J_{R}\left(2 J_{R}+1\right)(\ell+\varepsilon)\left(\Delta+2 J_{R} \varepsilon+\ell+2\right)} \\
f_{\Delta+1, \ell-1}^{J_{R}} & =(-1) \frac{J_{12} J_{34} \ell\left(\Delta-\ell+2 J_{R} \varepsilon\right)\left(\Delta-\ell-2\left(J_{R}+1\right) \varepsilon\right)}{2 J_{R}\left(J_{R}+1\right)(\ell+\varepsilon)(\Delta-\ell-2 \varepsilon+2)(\Delta-\ell-2 \varepsilon)}, \\
f_{\Delta+1, \ell+1}^{J_{R}} & =(-1) \frac{J_{12} J_{34}(\ell+2 \varepsilon)\left(\Delta+\ell-2 J_{R} \varepsilon\right)\left(\Delta+\ell+2 \varepsilon\left(J_{R}+1\right)\right)}{2 J_{R}\left(J_{R}+1\right)(\Delta+\ell)(\Delta+\ell+2)(\ell+\varepsilon)}, \\
f_{\Delta+1, \ell-1}^{J_{R}+1} & =\frac{A^{-} B^{-} \ell\left(\Delta-\ell-2 \epsilon\left(J_{R}+1\right)\right)}{2\left(J_{R}+1\right)\left(2 J_{R}+1\right)(\ell+\epsilon)\left(\Delta-\ell+2-2 \epsilon\left(J_{R}+2\right)\right)}, \\
f_{\Delta+1, \ell+1}^{J_{R}+1} & =\frac{A^{-} B^{-}(\ell+2 \epsilon)\left(\Delta+\ell-2 \epsilon J_{R}\right)}{2\left(J_{R}+1\right)\left(2 J_{R}+1\right)(\ell+\epsilon)\left(\Delta+\ell+2-2 \epsilon\left(J_{R}+1\right)\right)} .
\end{aligned}
$$

At higher level, the expressions become increasingly complex. For instance, the simplest expressions at level two are

$$
\begin{aligned}
f_{\Delta+2, \ell}^{J_{R}-2} & =\frac{\left(J_{12}+J_{R}-1\right)\left(J_{12}+J_{R}\right)\left(J_{34}+J_{R}-1\right)\left(J_{34}+J_{R}\right)\left(\Delta+2 J_{R} \varepsilon-\ell\right)\left(\Delta+2\left(J_{R}+1\right) \varepsilon+\ell\right)}{4\left(J_{R}-1\right) J_{R}\left(2 J_{R}-1\right)\left(2 J_{R}+1\right)\left(\Delta+\ell+2+2 \varepsilon J_{R}\right)\left(\Delta-\ell+2+2 \varepsilon\left(J_{R}-1\right)\right)}, \\
f_{\Delta+2, \ell}^{J_{R}+2} & =\frac{A^{-}\left(A^{-}+1\right) B^{-}\left(B^{-}+1\right)\left(\Delta-2 J_{R} \varepsilon+\ell\right)\left(\Delta-2 J_{R} \varepsilon-\ell-2 \varepsilon\right)}{16\left(J_{R}+1\right)_{2}\left(J_{R}+\frac{1}{2}\right)_{2}\left(\Delta-2 J_{R} \varepsilon-\ell-4 \varepsilon+2\right)\left(\Delta-2 J_{R} \varepsilon+\ell-2 \varepsilon+2\right)} .
\end{aligned}
$$


Note that many of them are related to one another by the relation explained in section 4 . An exhaustive list of the coefficients for the long multiplets can be found in the supplementary material.

Open Access. This article is distributed under the terms of the Creative Commons Attribution License (CC-BY 4.0), which permits any use, distribution and reproduction in any medium, provided the original author(s) and source are credited.

\section{References}

[1] M. Lüscher, Operator product expansions on the vacuum in conformal quantum field theory in two spacetime dimensions, Commun. Math. Phys. 50 (1976) 23 [INSPIRE].

[2] G. Mack, Convergence of operator product expansions on the vacuum in conformal invariant quantum field theory, Commun. Math. Phys. 53 (1977) 155 [INSPIRE].

[3] F.A. Dolan and H. Osborn, Conformal four point functions and the operator product expansion, Nucl. Phys. B 599 (2001) 459 [hep-th/0011040] [INSPIRE].

[4] F.A. Dolan and H. Osborn, Conformal partial waves and the operator product expansion, Nucl. Phys. B 678 (2004) 491 [hep-th/0309180] [INSPIRE].

[5] R. Rattazzi, V.S. Rychkov, E. Tonni and A. Vichi, Bounding scalar operator dimensions in 4D CFT, JHEP 12 (2008) 031 [arXiv:0807.0004] [INSPIRE].

[6] S. Rychkov, EPFL lectures on conformal field theory in $\geq 3$ dimensions, Briefs in Physics, Springer, Germany (2016), arXiv:1601.05000.

[7] D. Simmons-Duffin, The Conformal Bootstrap, in the proceedings of the Theoretical Advanced Study Institute in Elementary Particle Physics: New Frontiers in Fields and Strings (TASI 2015), June 1-26, Boulder, U.S.A. (2015), arXiv:1602.07982 [INSPIRE].

[8] D. Poland, S. Rychkov and A. Vichi, The conformal bootstrap: theory, numerical techniques and applications, Rev. Mod. Phys. 91 (2019) 015002 [arXiv:1805.04405] [INSPIRE].

[9] S.M. Chester, Weizmann lectures on the numerical conformal bootstrap, arXiv:1907.05147 [INSPIRE].

[10] S. Ferrara, A.F. Grillo and R. Gatto, Manifestly conformal covariant operator-product expansion, Lett. Nuovo Cim. 2S2 (1971) 1363 [INSPIRE].

[11] A.A. Migdal, Conformal invariance and bootstrap, Phys. Lett. B 37 (1971) 386.

[12] S. Ferrara, A.F. Grillo, G. Parisi and R. Gatto, Covariant expansion of the conformal four-point function, Nucl. Phys. B 49 (1972) 77 [Erratum ibid. B 53 (1973) 643] [InSPIRE].

[13] S. Ferrara, A.F. Grillo and R. Gatto, Tensor representations of conformal algebra and conformally covariant operator product expansion, Annals Phys. 76 (1973) 161 [INSPIRE].

[14] A.M. Polyakov, Nonhamiltonian approach to conformal quantum field theory, Zh. Eksp. Teor. Fiz. 66 (1974) 23 [INSPIRE].

[15] S. Ferrara, R. Gatto and A.F. Grillo, Properties of partial wave amplitudes in conformal invariant field theories, Nuovo Cim. A 26 (1975) 226 [INSPIRE].

[16] S. Ferrara, R. Gatto and A.F. Grillo, Positivity restrictions on anomalous dimensions, Phys. Rev. D 9 (1974) 3564 [INSPIRE]. 
[17] S. Ferrara, A.F. Grillo, R. Gatto and G. Parisi, Analyticity properties and asymptotic expansions of conformal covariant green's functions, Nuovo Cim. A 19 (1974) 667 [INSPIRE].

[18] V.K. Dobrev et al., Harmonic analysis on the n-dimensional Lorentz group and its application to conformal quantum field theory, Lect. Notes Phys. 63 (1977) 1 [INSPIRE].

[19] M. Buican, J. Hayling and C. Papageorgakis, Aspects of superconformal multiplets in $D>4$, JHEP 11 (2016) 091 [arXiv: 1606.00810] [INSPIRE].

[20] C. Cordova, T.T. Dumitrescu and K. Intriligator, Multiplets of superconformal symmetry in diverse dimensions, JHEP 03 (2019) 163 [arXiv:1612.00809] [INSPIRE].

[21] S.M. Chester, J. Lee, S.S. Pufu and R. Yacoby, Exact correlators of BPS operators from the 3d superconformal bootstrap, JHEP 03 (2015) 130 [arXiv:1412.0334] [INSPIRE].

[22] P. Liendo and C. Meneghelli, Bootstrap equations for $\mathcal{N}=4$ SYM with defects, JHEP 01 (2017) 122 [arXiv:1608.05126] [INSPIRE].

[23] C. Bee et al., The $\mathcal{N}=2$ superconformal bootstrap, JHEP 03 (2016) 183 [arXiv:1412.7541] [INSPIRE].

[24] M. Lemos and P. Liendo, Bootstrapping $\mathcal{N}=2$ chiral correlators, JHEP 01 (2016) 025 [arXiv: 1510.03866] [INSPIRE].

[25] C.-M. Chang, M. Fluder, Y.-H. Lin and Y. Wang, Spheres, charges, instantons and bootstrap: a five-dimensional odyssey, JHEP 03 (2018) 123 [arXiv: 1710.08418] [INSPIRE].

[26] C.-M. Chang and Y.-H. Lin, Carving out the end of the world or (superconformal bootstrap in six dimensions), JHEP 08 (2017) 128 [arXiv: 1705.05392] [INSPIRE].

[27] N. Bobev, E. Lauria and D. Mazac, Superconformal blocks for SCFTs with eight supercharges, JHEP 07 (2017) 061 [arXiv:1705. 08594] [INSPIRE].

[28] C. Beem et al., Infinite chiral symmetry in four dimensions, Commun. Math. Phys. 336 (2015) 1359 [arXiv: 1312 .5344] [inSPIRE].

[29] N.B. Agmon, S.M. Chester and S.S. Pufu, Solving M-theory with the conformal bootstrap, JHEP 06 (2018) 159 [arXiv:1711.07343] [INSPIRE].

[30] N.B. Agmon, S.M. Chester and S.S. Pufu, The M-theory archipelago, arXiv:1907.13222 [INSPIRE].

[31] F.A. Dolan and H. Osborn, Superconformal symmetry, correlation functions and the operator product expansion, Nucl. Phys. B 629 (2002) 3 [hep-th/0112251] [INSPIRE].

[32] F.A. Dolan, L. Gallot and E. Sokatchev, On four-point functions of 1/2-BPS operators in general dimensions, JHEP 09 (2004) 056 [hep-th/0405180] [INSPIRE].

[33] M. Nirschl and H. Osborn, Superconformal Ward identities and their solution, Nucl. Phys. B 711 (2005) 409 [hep-th/0407060] [INSPIRE].

[34] N. Bobev, S. El-Showk, D. Mazac and M.F. Paulos, Bootstrapping SCFTs with four supercharges, JHEP 08 (2015) 142 [arXiv:1503.02081] [INSPIRE].

[35] N. Seiberg and E. Witten, Comments on string dynamics in six-dimensions, Nucl. Phys. B 471 (1996) 121 [hep-th/9603003] [INSPIRE].

[36] O.J. Ganor and A. Hanany, Small $E_{8}$ instantons and tensionless noncritical strings, Nucl. Phys. B 474 (1996) 122 [hep-th/9602120] [INSPIRE].

[37] D. Gaiotto, $N=2$ dualities, JHEP 08 (2012) 034 [arXiv:0904.2715] [INSPIRE]. 
[38] J.J. Heckman and C. Vafa, Fine tuning, sequestering and the swampland, Phys. Lett. B 798 (2019) 135004 [arXiv: 1905.06342] [InSPIRE].

[39] W. Nahm, Supersymmetries and their representations, Nucl. Phys. B 135 (1978) 149 [INSPIRE].

[40] F.A. Dolan and H. Osborn, On short and semi-short representations for four-dimensional superconformal symmetry, Annals Phys. 307 (2003) 41 [hep-th/0209056] [INSPIRE].

[41] F.A. Dolan and H. Osborn, Conformal partial waves: further mathematical results, arXiv:1108.6194 [INSPIRE].

[42] S. Minwalla, Restrictions imposed by superconformal invariance on quantum field theories, Adv. Theor. Math. Phys. 2 (1998) 783 [hep-th/9712074] [INSPIRE].

[43] S. Ferrara and E. Sokatchev, Universal properties of superconformal OPEs for $1 / 2$ BPS operators in $3 \leq D \leq 6$, New J. Phys. 4 (2002) 2 [hep-th/0110174] [INSPIRE].

[44] V.K. Dobrev and V.B. Petkova, All positive energy unitary irreducible representations of extended conformal supersymmetry, Phys. Lett. B 162 (1985) 127.

[45] V.K. Dobrev and V.B. Petkova, On the group theoretical approach to extended conformal supersymmetry: classification of multiplets, Lett. Math. Phys. 9 (1985) 287 [INSPIRE].

[46] V.K. Dobrev and V.B. Petkova, Group theoretical approach to extended conformal supersymmetry: function space realizations and invariant differential operators, Fortsch. Phys. 35 (1987) 537 [INSPIRE].

[47] J. Bhattacharya, S. Bhattacharyya, S. Minwalla and S. Raju, Indices for superconformal field theories in 3, 5 and 6 dimensions, JHEP 02 (2008) 064 [arXiv:0801.1435] [INSPIRE].

[48] C. Cordova, T.T. Dumitrescu and K. Intriligator, Deformations of superconformal theories, JHEP 11 (2016) 135 [arXiv:1602.01217] [INSPIRE].

[49] M. Hogervorst and S. Rychkov, Radial coordinates for conformal blocks, Phys. Rev. D 87 (2013) 106004 [arXiv:1303.1111] [INSPIRE].

[50] M.S. Costa, T. Hansen, J. Penedones and E. Trevisani, Radial expansion for spinning conformal blocks, JHEP 07 (2016) 057 [arXiv: 1603.05552] [INSPIRE].

[51] F. Kos, D. Poland and D. Simmons-Duffin, Bootstrapping the $O(N)$ vector models, JHEP 06 (2014) 091 [arXiv: 1307.6856] [INSPIRE].

[52] F. Kos, D. Poland and D. Simmons-Duffin, Bootstrapping mixed correlators in the $3 D$ Ising model, JHEP 11 (2014) 109 [arXiv:1406.4858] [INSPIRE].

[53] J. Penedones, E. Trevisani and M. Yamazaki, Recursion relations for conformal blocks, JHEP 09 (2016) 070 [arXiv: 1509.00428] [INSPIRE].

[54] R. Rattazzi, S. Rychkov and A. Vichi, Bounds in $4 D$ conformal field theories with global symmetry, J. Phys. A 44 (2011) 035402 [arXiv: 1009.5985] [InSPIRE].

[55] S.M. Chester, J. Lee, S.S. Pufu and R. Yacoby, The $\mathcal{N}=8$ superconformal bootstrap in three dimensions, JHEP 09 (2014) 143 [arXiv: 1406.4814] [INSPIRE].

[56] A.L. Fitzpatrick et al., Covariant approaches to superconformal blocks, JHEP 08 (2014) 129 [arXiv: 1402.1167] [INSPIRE].

[57] F. Kos, D. Poland, D. Simmons-Duffin and A. Vichi, Bootstrapping the $O(N)$ archipelago, JHEP 11 (2015) 106 [arXiv:1504.07997] [INSPIRE]. 
[58] F. Kos, D. Poland, D. Simmons-Duffin and A. Vichi, Precision islands in the Ising and $O(N)$ models, JHEP 08 (2016) 036 [arXiv: 1603.04436] [INSPIRE].

[59] Z. Li and N. Su, Bootstrapping mixed correlators in the five dimensional critical $O(N)$ models, JHEP 04 (2017) 098 [arXiv: 1607.07077] [INSPIRE].

[60] D. Li, D. Meltzer and A. Stergiou, Bootstrapping mixed correlators in $4 D \mathcal{N}=1$ SCFTs, JHEP 07 (2017) 029 [arXiv: 1702.00404] [INSPIRE].

[61] J. Rong and N. Su, Bootstrapping minimal $\mathcal{N}=1$ superconformal field theory in three dimensions, arXiv: 1807.04434 [INSPIRE].

[62] S.R. Kousvos and A. Stergiou, Bootstrapping mixed correlators in three-dimensional cubic theories, SciPost Phys. 6 (2019) 035 [arXiv:1810.10015] [INSPIRE].

[63] C. Beem, M. Lemos, L. Rastelli and B.C. van Rees, The $(2,0)$ superconformal bootstrap, Phys. Rev. D 93 (2016) 025016 [arXiv: 1507.05637] [InSPIRE].

[64] J.J. Heckman, D.R. Morrison and C. Vafa, On the classification of $6 D$ SCFTs and generalized ADE orbifolds, JHEP 05 (2014) 028 [Erratum ibid. 06 (2015) 017] [arXiv: 1312.5746] [INSPIRE].

[65] J.J. Heckman, D.R. Morrison, T. Rudelius and C. Vafa, Atomic Classification of $6 D$ SCFTs, Fortsch. Phys. 63 (2015) 468 [arXiv:1502.05405] [INSPIRE].

[66] L. Bhardwaj, D.R. Morrison, Y. Tachikawa and A. Tomasiello, The frozen phase of F-theory, JHEP 08 (2018) 138 [arXiv: 1805.09070] [INSPIRE].

[67] J.J. Heckman and T. Rudelius, Top down approach to 6D SCFTs, J. Phys. A 52 (2019) 093001 [arXiv: 1805.06467] [INSPIRE].

[68] F. Baume, M. Fuchs and C. Lawrie, Solving superconformal matter: a $6 D$ bootstrap story, to appear. 